\title{
Algebraic Frameworks for Measurement in the Natural Sciences
}

\author{
Invited paper
}

\author{
Zoltan Domotor*
}

Departments of Philosophy, Biochemistry and Biophysics, University of Pennsylvania, Philadelphia, PA 19104, USA

The goals of this paper fall into three related areas: (i) we present an overview of a universal algebraic paradigm in which measurement specialists can construct formal models of measurement in a unified manner and systematically reason about a large class classical measurement operations, (ii) we construct convenient von Neumann quantity algebras and quantity-channels between them to represent measurements, and introduce the dual framework of state spaces and state-channels between them to investigate the statistical structure of measurements, and (iii) we provide several detailed examples that illustrate the power and versatility of algebraic approaches to measurement procedures.

Keywords: Discretization, duality, measurement operation, pointer quantity, quantity algebra, quantity-channel, state-channel, state space

\section{INTRODUCTION AND GENERAL BACKGROUND}

$\mathrm{T}$ HE MAIN purpose of this paper is to study a large class of classical measurement procedures in the frameworks of quantity algebras and quantity-channels, and in a dual measure-theoretic setting of state spaces and state-channels.

Measurement procedures are modeled at different levels of abstraction, reflecting the variety of options in isolating the empirical and statistical structures of interest that effectively characterize various measurement operations from a given perspective. In view of different conceptualizations, there are many competing models of measurement, and each model is limited by the assumptions made in its design. In this section we briefly describe three major theoretical approaches to measurement that enjoy significant prominence in various disciplines of applied science and engineering, and then move on to present the algebraic paradigm on which this paper is based.

In some ways, the most natural place to start is with the oldest representational theory of measurement (acronymed RTM), systematically developed by Krantz et al. in three volumes, starting with the expository volume [8]. In the tradition of empiricist methodology, the core idea of RTM is to treat measurement as a process of assigning numbers to certain qualitative attributes - instantiated by a given domain of stereotype empirical entities (objects, events, etc.) - in such a way that the comparative relation characterizing the attribute under consideration is faithfully represented by a corresponding order relation on numbers. In technical terms, measurement procedures are captured by various (embedding) homomorphisms from given empirical order structures to selected numerical order structures. ${ }^{1}$ This kind of

\footnotetext{
${ }^{*}$ Corresponding author: zdomotor@sas.upenn.edu

${ }^{1}$ For example, the length attribute of rods is characterized by the associated "shorter than" comparative relation or by its converse, the temperature of objects comes with the obvious "colder than" binary relation, and so forth. Here the important illustrating point is that upon introducing the mathematical shorthand $x \otimes y$ for "object $y$ is colder than object $x$ ", the definition of an embedding homomorphism $Q$ for temperature can be stated quite simply in
}

measurement-theoretic representation is shown in Figure 1 below, in which the homomorphism $Q$ between an empirical relational structure $\langle\mathscr{O}, \ominus\rangle$ and a numerical relational structure $\langle R,<\rangle$ is also known under the uninspired name "scale of measurement".

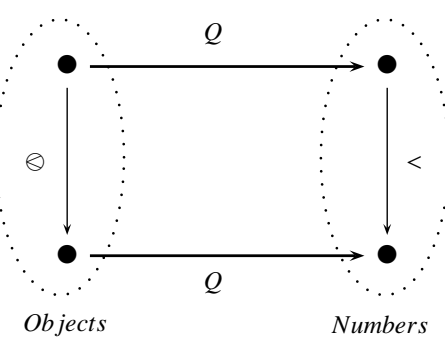

Fig. 1. Visualization of measurement as an order-preserving map from a given domain of objects $\mathscr{O}$ to that of numbers $\mathbb{R}$.

The assignment of numbers to degrees, levels or amounts of attributes instantiated by objects or events is fundamental, since it justifies the use of real numbers and real-valued functions in science. A crucial critical remark here is that the enlisted help of mathematics does not come to significantly more than the study of various types of scales of measurement, transformations between them, and some related criteria for empirical meaningfulness. Crucially, RTM's central measurement problem lies in ensuring the measurability of attributes or in other words the numerical representability of their empirical order structure. Recall that for an attribute to be measurable, it is necessary to provide a list of

the form of equivalence $x \ominus y \Longleftrightarrow Q(x)<Q(y)$ for all objects $x$ and $y$. In this way, the natural ordering of numbers faithfully emulates the temperaturebased ordering of objects. Reflecting this fact, $Q(x)$ is the temperature of object $x$ in the "scale" described by $Q$. An unfortunate aspect of RTM's homomorphisms is that they exist (in a strictly nonconstructive sense) only if the comparative relation $\ominus$ satisfies certain nontrivial axioms, such as the familiar transitivity condition: $(x \ominus y \& y \ominus z) \Longrightarrow x \ominus z$ for all entities $x, y$ and $z$. This general picture can be extended by adding compatible composition operations on objects and events or by considering an empirical ordering of pairs of objects. 
conditions (a.k.a. axioms), governing the attribute's comparative relation, that is sufficient for the existence of an embedding homomorphism from the empirical order structure to the numerical order structure. It is disenchanting to learn that in many practical cases the decisive axioms (e.g., the socalled Archimedean axiom that excludes infinitesimals) are of a highly theoretical nature, known to be difficult to test in the attribute's domain of instantiating objects. Moreover, in some applications of RTM it is truly hard to find the correct list of empirical conditions that grants the existence of a representing homomorphism. For example, it is far from clear what axioms should a measurement specialist choose for measuring the level of violence depicted in TV movies, in order to grant the existence of a homomorphism that provides meaningful and usable numbers for the occurrence of violent acts in motion pictures.

As practical mathematical tools, RTM's models of measurement tend to operate at a lamentably high level of abstraction. For example, RTM's axiomatic formulation of an equal-arm balance measurement cannot distinguish mass from weight. Along similar lines, RTM's setup developed for the traditional direct-comparison based measurement of length of rods by placing alongside a ruler and stepping it off against the rod under consideration, does not single out this collinear type of measurement from some other, say, nonstandard orthogonal measurements, employing the Pythagorean theorem. This tells us that incorporating specific modes of measurement into RTM's models is an extremely problematic enterprise. Since extensive quantities are axiomatized basically in the same way, it is not clear exactly which quantity is being measured. Another important practical point is that in measuring length, RTM relies on the existence of highly idealized straight-line rigid bodies, yet the length attribute is instantiated also by ropes, rubber bands, and a host of other curved real-world nonrigid objects. RTM's measurement models do not make a sharp distinction between establishing the value of a given attribute by measurement vs. by theory. For example, since temperature is a theoretical thermodynamical quantity (defined by the partial derivative of the system's internal energy over entropy) governed by thermodynamical laws, its values cannot be determined effectively by fundamental measurement, based on the "colder than" qualitative relation. Instead, temperature is commonly measured indirectly, say, by a thermometer with a mercury column, whose length is linearly related to temperature. The point here, of course, is that a realistic measurement of a target attribute $Q$ (instantiated by an object) employs a second (directly observable) pointer attribute $Q^{\uparrow}$ of a measuring instrument, whose values are nomologically related to those of $Q$ by an equation of the form $Q^{\uparrow}=\mathbf{F}(Q){ }^{2}$ Thus, to obtain the value of $Q$ from a theory is one thing and to acquire $Q$ 's

\footnotetext{
${ }^{2}$ For example, resistance temperature devices rely on the simplified empirical resistance law $\mathrm{R}=\mathrm{R}_{0}(1+\alpha \cdot \mathrm{T})$, stating that a platinum wire's resistance $R$ linearly varies with its ambient temperature $T$, where $R_{0}$ denotes the wire's resistance in a melting ice and $\alpha$ stands for the platinum's material constant.
}

value by measurement is yet another. Because the attributes of scientific interest stand in crucial lawful relationships with other attributes, their measurements are practically always indirect or derived. It must be recognized that RTM is not able to serve the complex needs of engineering applications, scientific model development or theory validation. Therefore, despite its prominence in philosophy of science, mathematical psychology and economics, RTM finds itself in a minority position within the natural sciences.

The strongest criticisms of RTM concentrate on the limited applicability of RTM's measurement models in engineering and applied sciences. For example, RTM typically does not include in its measurement models the all-important causeeffect and other law-like relations between measurable attributes, it lacks concrete representations of measuring procedures, instruments and their dynamical interactions, and it is not tailored for a statistical description of measurement errors, noise, and uncertainties in general. Additional critical analysis of RTM may be found in [2].

The second approach to measurement processes is provided by the combined resources of mathematical systems and signal theories or more concretely, by the conceptual framework of algebraic signal processing theory of measurement, hereafter abbreviated as STM. As a by-product of classical systems and signal theories, STM is built around temporally (and spatially) varying smooth physical signals ${ }^{3}$ and their transformations thereon by man-made physical systems, including but not limited to sensors, transducers, filters, amplifiers, analog-digital converters ${ }^{4}$, and signal reconstruction (inversion) modules. At the heart of STM rests the reductionist idea that measurement processes are merely special cases of other processes studied in mathematical systems and signal theories. In engineering science, measurements of a target system's signal are described by joint families of differential equations with constant coefficients in a time or state domain. As is well known, the traditional input-state-output framework of systems theory is extremely well developed and is widely accepted in the engineering community. The basic formal modeling methodology of STM consists of associating with each physical system a suitable transfer operator or a transfer matrix (obtained from the representing differential equations) that sends the system's input signals to its output signals, mirroring - modulo admissible errors - the system's actual signal processing behavior in a designated range.

Within STM, measurement is understood to involve a continuous-time detection, processing and presentation of information carried by the signals of interest that are fed by the target system into a serially connected chain of several separate elements. In simple situations, a measuring instrument consists of a front-end sensor and a cascaded tail-end

\footnotetext{
${ }^{3}$ Intuitively, signals are understood to be temporally or spatially varying physical quantities, used to convey information about designated physical phenomena. Signals are represented by functions on a time, space or related domains. The most prominent examples of variation are smooth, continuous and discrete temporal changes in signal values.

${ }^{4}$ For practical estimation algorithms in signal sampling see [5].
} 
signal inversion or reconstruction unit. ${ }^{5}$ A sensor is a systeminstrument interface hardware, serving as a crucial component of most measuring instruments. It converts the information of interest (e.g., about the degree of temperature, pressure, and so forth) carried by the target system's unknown output signal (i.e., time-dependent measurand) $Q(t)$ into a correlated information, carried by the sensor's electrical, optical or some other kind of output signal. Since the design of sensors is based on fairly well-established physical principles, the description of their behavior is given by the technical resources of applied physics.

It is not generally realized that in STM the sensor's output signal $Q^{\prime}(t)$ is just a raw intermediary that has to be fed into a reconstructor (inversion) module in order to be able to obtain the terminal signal $\widehat{Q}(t)$ of interest which optimally estimates or approximates the system's unknown measurand $Q(t)$ with measurement error $E_{Q}(t)={ }_{d f}|\widehat{Q}(t)-Q(t)|$. In fact, in ideal measurements the estimate $\widehat{Q}(t)$ is obtained from the measurand $Q(t)$ by the composite transformation $\widehat{Q}(t)=\mathbf{F}^{-1} \circ \mathbf{F}(Q(t))$, where $\mathbf{F}$ denotes the sensor's transfer operator and $\mathbf{F}^{-1}$ stands for the reconstructor's input-output operator. Although in the majority of measuring devices the representing transfer operator $\mathbf{F}$ is not invertible, here we assume that the reconstruction unit is designed in such a way that its transfer operator can be viewed as a generalized left inverse of $\mathbf{F}$ that solves the so-called inverse problem. ${ }^{6}$ Figure 2 shows the block diagram of a signal flow from the target system to a measuring instrument and the physical arrangement of instrument modules.

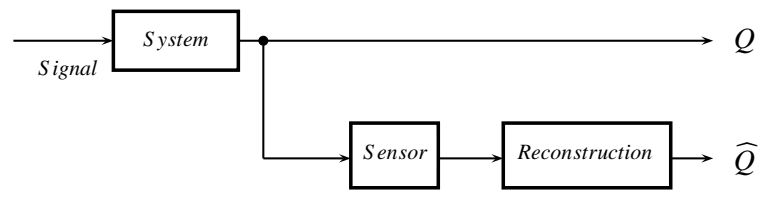

Fig. 2. Signal flow diagram of a simple measurement

In most commercial instruments, the reconstructor is just an analog or digital information processing module that is programmed or calibrated to generate the "reconstructed" signal $\widehat{Q}(t)$ from the sensor's output signal $Q^{\prime}(t)$. It is important to bear in mind that in the sensory and reconstruction transformations of signals there is no reference to numbers. The measuring instrument's output signal $\widehat{Q}(t)$ is usually fed automatically into a feedback control system or is transmitted to a

\footnotetext{
${ }^{5}$ Here the notion of reconstruction is similar to the one introduced by $\mathrm{R}$. Z. Morawski in [9].

${ }^{6}$ For example, if the information carried by the signals is described by a Gaussian probability density function, then the inverse problem is usually solved by the method of least squares that determines the signal's least squares estimator. For concrete applications see [7]. It is easy to see that if an incrementally linear sensor is described by the equation $Q^{\prime}(t)=2 \cdot Q(t)+3$, then the inversion module is characterized by the equation $Q(t)=\frac{1}{2}\left(Q^{\prime}(t)-3\right)$.
}

remote place, where it is recorded in a computer for additional processing or storage, without any direct (human) observer intervention. There is also a less common option in which the signal is sent to a display unit.

Not surprisingly, STM has not been the subject of much cirtitical scrutiny, because (i) signals and systems theories possess solid mathematical foundations and enjoy an uncommonly wide range of applications (see in particular [11]), and (ii) measurement engineers and practicing scientists are seldom concerned with foundational issues in spite of the fact that the study of measurement has always been impeded to various degrees by inadequate treatments of its foundational aspects. We shall now move on to provide a brief assessment of STM that is limited to (a) the nature of STM's models of measurement, and (b) to STM's interpretation of measurement.

First, one unfortunate aspect of traditional systems theory (and its applications in STM) is that it treats inputs and outputs as intrinsic features of systems. As noted earlier, in the inputstate-output systems-theoretic framework, the typical interaction between two systems is handled by placing the systems in series between input and output signals, forming appropriate signal algebras $\mathfrak{A}$ and $\mathfrak{A}^{\prime}$ respectively, as shown schematically in Figure 3.

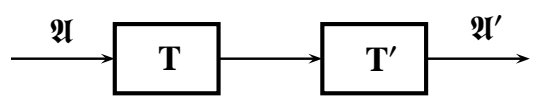

Fig. 3. Traditional transfer operator (matrix) description of cascaded systems.

Since the resulting transfer operator representing the cascaded total system is given by the composition $\mathbf{T}^{\prime} \circ \mathbf{T}$ of operators modeling the respective component systems, we can quickly conclude that STM's measurement models have the equational operator form $\widehat{Q}(t)=\mathbf{F}_{n} \circ \cdots \circ \mathbf{F}_{2} \circ \mathbf{F}_{1}(Q(t))$, where the sequence $\mathbf{F}_{1}, \mathbf{F}_{2}, \cdots \mathbf{F}_{n}$ refers to the the transfer operators representing the measuring instrument's serially connected components with input measurand $Q(t)$ and its output estimate $\widehat{Q}(t)$.

Second, since STM's models of measurement rely on special representations of signals, using Fourier, Laplace, bilateral $\mathrm{Z}$ and other transformations that do not automatically generalize to noncommutative signal algebra frameworks, there are no readily accessible formal tools for modeling quantum measurement.

We end our assessment of STM's models with a brief digression in which we consider an alternative to classical systems theory that is a dual or a mirror image of the inputoutput transfer operator approach, recalled above. ${ }^{7}$ It is widely known that the dynamical behaviors of most systems encountered in physics, chemistry, biology and engineering

\footnotetext{
${ }^{7}$ The formal framework for measurement advocated in this paper is a variant of this dual perspective.
} 
can be effectively characterized by (ordinary, partial, timedelay, etc.) differential or difference equations, without separating the pertinent signals into intrinsically given 'input' and 'output' types. The reader is urged to consider the dynamical behavior of a simple pendulum or that of a planet in our solar system on which various measurements are regularly performed and ask "What is the intrinsic input signal of a pendulum and what is the standard output signal of the planet Mars?" Although everybody recognizes that the inputoutput framework of systems theory enjoys wide ranging applications, for the purposes of measurement it is necessary to cast systems-theoretic models in a considerably more general setting that is capable of a highly flexible representation of any measurement operation whatsoever, performed on any target system studied in the natural sciences and engineering. A big step in this direction came with the developments of algebraic and behavioral systems theories that are based on signal algebras (or signal spaces).

The essential idea is to proceed dually to the traditional systems theory and associate with each system an appropriate signal algebra $\mathfrak{A}$ that provides a formal ambience for all possible law-like relations between temporally varying signals and for the target system's possible dynamical behaviors. ${ }^{8}$ The dual algebraic approach to systems is not limited to systems lacking inputs or outputs. As shown in Figure 4, with the help of tensor product algebras, the algebraic framework can also handle systems that require a decomposition of signals into input and output types.

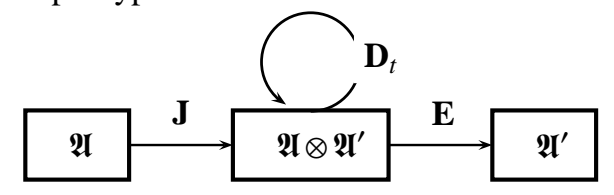

Fig. 4. Signal algebra description of cascaded systems.

In the case of two interacting systems, say, a target system and a measuring instrument, each constituent system is described by an appropriate signal algebra $\mathfrak{A}$ and $\mathfrak{A}^{\prime}$ respectively, and the combined system is characterized by the product signal algebra $\mathfrak{A} \otimes \mathfrak{H}^{\prime}$, generated by coupled or entangled signals. We know that for a dynamical measurement to take place, there must be a physical interaction between the measurand's system and the measuring instrument that outputs a measurement result at the end of the joint dynamical evolution of the total system, given suitable initial conditions. Such interactions are modeled by tensor product signal algebras. Now, the signal algebra-based approach recovers the inputoutput relations from the tensor product signal algebra with the help of (i) a natural embedding algebra homomorphism $\mathbf{J}$, and (ii) a product-type expectation map $\mathbf{E}$. The time evolution of the joint system-plus-apparatus signal is represented

\footnotetext{
${ }^{8}$ Note that the boxes are now annotated with the names of signal algebras. In many engineering applications, signal algebras are usually specified by structured sets of smooth real-valued functions defined on a time domain, e.g., the algebra $\mathbf{C}^{\infty}(\mathbb{R})$ of smooth (i.e., infinitely differentiable) real-valued functions on the continuum time domain $\mathbb{R}$ of reals.
}

by law-based time-indexed automorphisms $\mathbf{D}_{t}$ of the product signal algebra.

Finally, notice that the algebraic framework capitalizes on ideas that make it suitable also for modeling passive and static measurements, in which the interaction is passive and time or space parameters are not included. As widely known, the simplest types of static deterministic measurements (e.g., length measurement by meter sticks, volume measurement by graduated measuring cups, granularity measurement by reference materials, etc.) are based on the principle of direct comparison, in which the quantity or signal to be measured is directly related to a measuring device's pointer quantity, which is carefully calibrated against the measurand's sampled values, without any explicit reference to dynamical interaction or temporal changes. As sketched in Figure 5, in elementary nonideal measurements, a measuring instrument's algebra (usually generated by its pointer quantity) $\mathfrak{A}$ is mapped by a homomorphism $\mathbf{M}$ to the target system's algebra $\mathfrak{A}^{\prime}$ in such a way that $\mathbf{M}$ sends the pointer quantity to the measurand's best estimate in $\mathfrak{H}^{\prime}$.

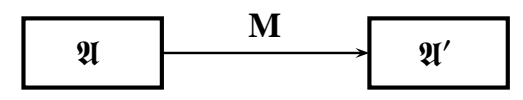

Fig. 5. Algebraic description of a static measurement, involving a target system and a measuring device.

It should be carefully noted that the homomorphism $\mathbf{M}$ goes from the instrument's algebra $\mathfrak{A}$ to the system's algebra $\mathfrak{I}^{\prime}$, because of the fact that the measurand's estimate is inferred from meter reading.

Returning from our digression on algebraic systems theory, we now make a few comments regarding STM's interpretation of measurement. On STM's signal-theoretic interpretation, measurement is basically a dynamical signal processing enterprise, whose purpose is to output the measured signal's estimate that meets the needs of intended applications in the world of control systems. Since the numerical values of signals are of secondary importance, measurement is not directed at making a contribution to the design and validation of scientific theories. Moreover, because STM is confined mainly to continuous-time dynamical measurements of signals, static and passive measurements do not play any role in it. Related to this, pointer quantities and their relationships to measurands are not included in STM's measurement models. In contrast to the above, classical measurement is commonly thought of as an empirical procedure, involving the measurand's target system that is dynamically coupled with a measuring instrument in a such a way that after having reached their stable joint entangled state, the instrument's needle will point to an ink mark of a numeral on its scale that is strongly related to the measurand's value. Last but not least, STM's measurement models do not exploit the mathematical duality between quantities and physical states that is of the highest importance in all approaches to measurement. Indeed, the 
simultaneously-present quantities (signals) are necessary for reasoning about the underlying target system and one-at-atime states render the possible ways in which the system fixes the values of its quantities.

The third approach to measurement we shall advocate here investigates measurement procedures by the powerful conceptual tools of algebraic analysis, such as normed linear algebras and quantity-channels between them, and their dual counterparts, formed by state spaces and state-channels. ${ }^{9}$ These algebraic-analytic structures will enable us to provide explicit descriptions of a large variety of measurements. In keeping with the terminology adopted above, we shall refer to the third approach as an algebraic theory of measurement (or simply ATM).

For the approach we are going to adopt in this paper, it is appropriate to view measurement as a physical operation performed on a target natural system that yields partial information about the extant value of the system's selected quantitative property. As mentioned in less detail earlier, measurement operations are implemented by physical interactions between a natural system under consideration and a measuring instrument, involving a calibrated pointer quantity that enables the experimenter to deduce the best estimate or approximation (limited by its accuracy, sensitivity and resolution) of the extant value of the system's quantity to be measured, commonly called the measurand, to which the pointer quantity is dynamically correlated during the interaction. Simply, known dynamical changes in the instrument are used to estimate (within a specified range) the unknown values of the coupled target system's measurand. In this way, certain (usually man-made) designated systems are used to tell us something informative about some other systems in the world. The amount of information provided by a measurement procedure depends on the strength of the underlying deterministic or stochastic linkage, specified by the dynamical coupling between the target system and measuring instrument.

Within ATM, a (physical, chemical, etc.) quantity ${ }^{10}$ is a formal entity ascribed to the target system's attribute of interest, whose numerical values encode the attribute's qualitative degree or amount possessed by the system at various moments of time. Epistemically, quantities are the observer's decisive information-gathering entities about the system's behavior. Therefore, as a quantity deployer, the experimenter queries the target system with various measurements of quantities. Importantly, as was mentioned before, the result of measurement of a target system's measurand depends on the system's extant physical state, and of course the system is pre-

\footnotetext{
${ }^{9}$ A linear algebra over the scalar field $\mathbb{R}$ of real or complex $\mathbb{C}$ numbers is a set which is both a ring and a linear (vector) space. A normed algebra is an algebra which is also a normed linear space. A norm is needed for the limit properties of certain sequences of quantities.

${ }^{10}$ Since in practice measurement involves several processing stages, starting with sensors, amplifiers and transducers, and ending with reconstruction, display and storage units, signal theorists have replaced the term quantity by the term signal that serves well in STM's approach to measurement. We shall use the term quantity because it is more general and is well established in the natural sciences.
}

sumed to have the capacity to be in different states without losing its systems-theoretic identity.

States encapsulate the totality of the system's internal conditions, i.e., the way the system is at various instants of time. As alluded to earlier, the most important feature of quantities is their dual relationship to states. The target system's state fully determines the values of the system's characterizing quantities and conversely, at any given time the quantity values collectively specify the system's state, i.e., the system's physical mode of being at that time. In the case of systems studied in statistical mechanics such state specifications are unmistakably statistical in nature.

Since at each time the target system is in a definite state, by the above-mentioned duality any quantity associated with the system must have a concrete numerical value, even if the experimenter does not know what it is. The nonempty set of possible values of a quantity $Q$ is commonly referred to as its spectrum or value space and is accordingly denoted by $\operatorname{Spec}(Q)$. The simplest nontrivial quantities are Boolean or two-valued, meaning with "no" and "yes" values, formally specified by the numerical doubleton $\operatorname{set} \operatorname{Spec}(Q)=\{0,1\}$ and observed in binary measurements, such as green/red alarm system lights, circuit testers, and other one-bit detectors. A considerably larger subset of the set of real numbers $\mathbb{R}$ is used for the values of mass, charge, energy and other quantities associated with physical systems. Crucially, by classical idealization it is assumed that the a quantity's continuum spectrum remains meaningful also at the Planck scale, even if most of the spectral values can never be identified exactly by any actual measurement procedure. Speaking philosophically, in view of measurement errors, limited sensitivity and resolution, thermodynamical fluctuations in the measuring instrument and quantum uncertainties, measurement-based epistemology provides strictly less information about the target system than available in the presumed ontology of quantities. Put another way, in general, experimenters are not equipped to know the precise values of the target system's quantities and the system's actual states. Simply, measurement results come in finite bits, expressed by rational numbers. What is usually known about the target systems are some reasonable approximations or interval-estimates of certain quantities and values, and likewise states. It should also be mentioned that in considering the value of a quantity $Q$, e.g., the target system's magnitude of energy, by 'value' we mean the numerical value of the algebraic object $Q$ that encodes the amount of energy that exists objectively in the system, independently of whether or not $Q$ is measured.

We propose to model measurement procedures by relying on the following two basic conceptual ingredients: (i) a $d o$ main of reality comprised of systems and measurement procedures thereon, and (ii) a domain of algebraic models together with quantity-channels between them, intended to reason about the chosen domain of systems and associated measurement procedures. We now turn to a general investigation of systems, measuring instruments and their involvement in measurement operations. 


\section{MONOIDS OF SYSTEMS AND CHANNEL STRUCTURES OF MEASUREMENT}

In the domain of reality we have a class natural systems of interest (e.g., various pendulums, electric circuits, gases or fluids in vessels, particles, elastic solids, and so forth) that are at any instant of time in various modes of being (e.g., being in a state of equilibrium, being heated, being accelerated, and so on), classically referred to as states. In general, a natural system is any part of the physical world that can (at least in principle) be separated from its ambience, and treated as an empirically meaningful and autonomous object of investigation. For us, a system is an objectively existing physical thing-in-itself that can be accessed by observation and measurement. The temporal evolution of systems is investigated in terms of quantity changes, reflecting the changes in the target system's states.

On the basis of past experience we assume that systems of the same physical type can be combined into larger systems by suitable coupling. Thus, by taking $S$ and $S^{\prime}$ to be two systems of interest in a given class of systems $\mathbf{S}$ of the same (or compatible) physical type, the expression $S+S^{\prime}$ denotes the composite system in $\mathbf{S}$ of systems $\mathbf{S}$ and $\mathbf{S}^{\prime}$. For example, two pendulums can be coupled to form a double pendulum, an electric circuit can be coupled with a voltmeter or with another electric circuit, and so on. Depending on the type of systems, the composition operation may be realized in several physically different ways, including (but not limited to) the most familiar parallel and series configurations. The class of systems $\mathbf{S}$ also includes copies of the trivial system $\mathrm{O}$ (e.g., a perfect wire in the class of electric circuits, a pendulum with negligible arm size in the class of simple pendulums, etc.). We must immediately recognize the difficulty in specifying the composite system $\mathrm{S}+\mathrm{S}$. We ask bluntly, how does the experimenter realistically compose a system with itself? The answer: with an acknowledged element of idealization it is assumed that each system in $\mathbf{S}$ has an unlimited number of physical copies. If two systems $S$ and $S^{\prime}$ are copies of each other, i.e., if they are physically similar in all relevant respects (including behavior and quantitative properties of interest), then we say that they are physically equivalent and write $S \approx S^{\prime} .{ }^{11}$ Now it seems reasonable to set the composite system $S+S$ to be physically equivalent to $S+S^{\prime}$ with $S \approx S^{\prime}$ for some system $\mathrm{S}^{\prime}$ and treat the composition operation in a generalized way. That is to say, the composite $S+S^{\prime}$ is meant to be unique modulo the congruence relation $\approx$. In particular, for all systems $\mathbf{S}, \mathbf{S}^{\prime}$ and $\mathbf{S}^{\prime \prime}$ in $\mathbf{S}$ the following conditions are assumed to hold:

(i) Commutativity: $\mathrm{S}+\mathrm{S}^{\prime} \approx \mathrm{S}^{\prime}+\mathrm{S}$.

(ii) Associativity: $\left(\mathrm{S}+\mathrm{S}^{\prime}\right)+\mathrm{S}^{\prime \prime} \approx \mathrm{S}+\left(\mathrm{S}^{\prime}+\mathrm{S}^{\prime \prime}\right)$.

(iii) Identity: $\mathrm{S}+\mathrm{O} \approx \mathrm{S} \approx \mathrm{O}+\mathrm{S}$.

\footnotetext{
${ }^{11}$ From an empiricist point of view, in this case no experiment addressing the behaviors under consideration can be used to distinguish $S$ from $\mathrm{S}^{\prime}$.
}

Physical equivalence is definable in terms of a subsystem relation. We say that $S$ is a subsystem of $S^{\prime}$ and write $S \leqslant S^{\prime}$, provided that $S$ is a physically meaningful part of $S^{\prime}$ with its local states and quantities, satisfying the Monotonicity

(iv) $\mathrm{S} \leqslant \mathrm{S}^{\prime} \Longrightarrow \mathrm{S}+\mathrm{S}^{\prime \prime} \leqslant \mathrm{S}^{\prime}+\mathrm{S}^{\prime \prime}$, and Nullity

(v) $\mathrm{O} \preccurlyeq \mathrm{S}$ conditions.

In this way, systems of the same physical type form a generalized partially ordered monoid $\langle\mathbf{S}, \mathrm{O},+, \preccurlyeq\rangle$, in which the physical equivalence relation $S \approx S^{\prime}$ is defined by the conjunction $S \preccurlyeq S^{\prime} \& S^{\prime} \leqslant S$. The simplest example of a partially ordered monoid of systems is given by the sequence

$$
\mathrm{O} \leqslant \mathrm{S} \leqslant \mathrm{S}+\mathrm{S} \leqslant \mathrm{S}+\mathrm{S}+\mathrm{S} \leqslant \cdots
$$

of composite systems and their copies, where $S$ is any system, say, a simple pendulum or a single Newtonian particle moving in a spatial region. The notion of physically analogous systems is captured by suitable homomorphisms between partially ordered monoids of systems that uphold a physically meaningful transformation of quantities from one class of systems to another. There are several other operations on systems (e.g., the join of two systems), but we shall not consider them here.

In order to be able to effectively reason about systems and measurements thereon, ATM moves from a domain of reality of interest to the domain of algebraic models by associating with each system $\mathbf{S}$ in $\mathbf{S}$ an suitable quantity algebra $\mathfrak{A}_{\mathrm{S}}{ }^{12}$ and its accompanying convex space $\mathbf{S}\left(\mathfrak{H}_{\mathrm{S}}\right)$ of states. ${ }^{13}$ Although the technical discussion in the preceding paragraph may seem off our informal track, it does point us in the right direction. Concretely, it tells us that investigators can model the dynamical behaviors of systems universally, in terms of appropriately chosen quantity algebras and state spaces. We have already emphasized that a target system's quantity algebra determines and is determined by a state space. The type of duality we have in mind is captured by the 2-level channel diagram below.

Next, we extend the foregoing modeling idea of systems and their behaviors to modeling measurement actions that are also elements of the objectively existing domain of reality. For our present purposes we shall use the mapping symbolism $\mathscr{M}: \mathrm{S} \rightarrow \mathrm{M}$ to denote a given elementary measurement operation $\mathscr{M}$ that transfers information from the target system

\footnotetext{
${ }^{12}$ Here it seems appropriate to ask: Why should measurement specialists associate an entire algebra of quantities with each system under consideration? Because the system's possible behaviors cannot be satisfactorily described by a frugal list of basic characterizing quantities (e.g., voltage, current and resistance in the case of classical electric circuits). Complete descriptions of system behaviors require longer lists of derived (defined) quantities together with law-like relations between them, scale changes and limit operations. These constructions on quantities are naturally accommodated by normed algebras.

${ }^{13}$ These and other related mathematical notions are discussed in more detail in the next section.
} 
$S$ to a selected measuring instrument $M$. In the case of a joint measurement procedure (involving a transfer of information to two designated instruments) we write $\mathscr{M}: \mathrm{S} \rightarrow\left(\mathrm{M}+\mathrm{M}^{\prime}\right)$. A measurement operation involving a target system that is dynamically coupled with an instrument is symbolized by the map $\mathscr{M}: \mathrm{S}+\mathrm{M} \rightarrow \mathrm{M}$, and so forth. This notational system handles remarkably well all classical measurement operations arising in engineering and applied sciences.

By analogy with the above, we associate with each (static) elementary measurement operation $\mathscr{M}: \mathrm{S} \rightarrow \mathrm{M}$, belonging to the domain of reality under consideration, a suitable statechannel of the form

$$
\mathbf{S}\left(\mathfrak{A}_{\mathrm{S}}\right) \stackrel{\mathbf{M}^{*}}{\longrightarrow} \mathbf{S}\left(\mathfrak{\mathfrak { I }}_{\mathrm{M}}\right)
$$

that represents the information transfer from the target system's state space $\mathbf{S}\left(\mathfrak{A}_{S}\right.$ to the measuring instrument's state space $\mathbf{S}\left(\mathfrak{\Re}_{M}\right)$. In classical situations, a state-channel is basically a transition probability (a.k.a. Markov kernel) that sends each probability distribution in the system's state space to a unique probability distribution in the measuring instrument's pointer state space. This model of information transfer readily extends to vastly more general state spaces.

Since in a large part of this paper we shall be concerned with a dual methodology that models systems both in terms of their physical states and quantities, it is also important to bring quantity-channels into the picture. Henceforth, if $\mathbf{M}$ denotes a quantity-channel, we shall adopt the habit of marking the associated state-channel with an asterisk, as in $\mathbf{M}^{*}$.

In an exact parallel to state-channels, the crucial idea now is to associate with each (static) elementary measurement operation $\mathscr{M}: \mathrm{S} \rightarrow \mathrm{M}$ a quantity-channel of the form

$$
\mathfrak{A}_{M} \stackrel{\mathbf{M}}{\longrightarrow} \mathfrak{I}_{\mathrm{S}}
$$

that represents the inferential transfer of data from the measuring instrument's quantity algebra to the system's algebra. Formally, a quantity-channel is a unit-preserving positive linear map between quantity algebras that sends the instrument's pointer quantity to the measurand's best estimate quantity. A question can now be raised: Why are we using quantitychannels instead of algebra homomorphisms? Because algebra homomorphisms are unnecessarily too restrictive in modeling many measurement procedures. Moreover, all known measurement operations and all known temporal evolutions of dynamical systems are universally representable by appropriate quantity-channels between quantity algebras. The problem is that homomorphisms do not preserve the general quantity-state duality we need. However, as it turns out, quantity-channels (specified by unit-preserving, positive linear maps) uphold the duality requirement.

In order to bring the full force of quantity-state duality into play, we close this section by recalling a couple of diagrams that elucidate the intimate relationship between quantity-channels and state-channels. A parallel account of inference-to-the-best-estimator and information transfer is illustrated in the diagram of maps below: ${ }^{14}$

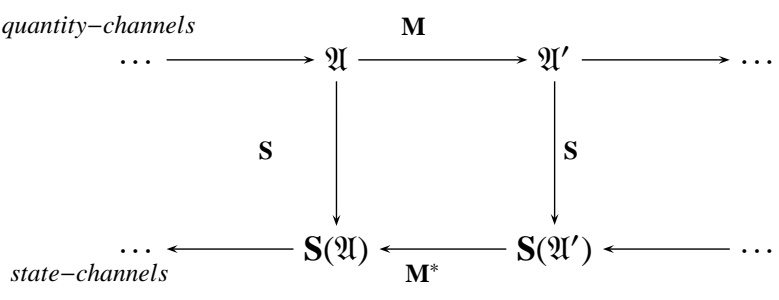

Experimenters investigating measurement methods can analyze and interpret measurement procedures in two complementary ways, based on

(i) State semantics: State-channels representing measurement operations are systematically interpreted in information-theoretic terms. For example, an ideal statechannel, bridging the target system and measuring instrument, transfers a maximal amount of information that faithfully determines the measurand's extant value or the probability thereof. On the other hand, nonideal statechannels obliterate the information transferred from the observed system to the instrument.

(ii) Quantity semantics: Quantity-channels, modeling measurement operations, are interpreted in inverse-problem inferential terms, where inferences from measurement data to the measurand's best estimates are viewed as estimation-algorithm results traversing the channel between the respective algebras of the measuring instrument and target system.

As one might guess from the foregoing semi-formal treatment of quantity-state duality, a quantity-channel $\mathbf{M}$ is oneto-one (injective) if an only if its corresponding state-channel $\mathbf{M}^{*}$ is onto (surjective). Likewise, a quantity-channel $\mathbf{M}$ is a surjective map precisely when $\mathbf{M}^{*}$ is injective. These and a large number of other properties of channels follow directly from a category-theoretic duality between quantity algebras and state spaces.

Unfortunately, since the preceding diagram is based entirely on general principles, it reveals very little information about the exact nature of state-quantity duality. For this reason, we need a commutative Chu space transformation $\operatorname{diagram}^{15}$

\footnotetext{
${ }^{14}$ To get the full picture of duality and to gain insight into the empirical meaning of the diagram of channels, we introduce a tensor category Qalg of quantity algebras (consisting of, e.g., Banach algebras or von Neumann algebras, discussed in Section 4) and quantity-channels between them. Next, we note that the opposite category Qalg ${ }^{o p}$ with its arrows reversed is equivalent to the category cProb of convex spaces of probability measures and transition probabilities between them. Now, the quantity-state duality is captured by the state functor $\mathbf{S}: \mathbf{Q a l g}^{o p} \longrightarrow \mathbf{c P r o b}$ and its left adjoint. By choosing quantity-channels as maps (and not algebra homomorphisms) we have implicitly imparted a crucial probabilistic structure to the objects of Qalg ${ }^{o p}$. What this means is that the objects of $\mathbf{Q a l g}^{o p}$ are representable by concrete probability spaces.

${ }^{15} \mathrm{Chu}$ spaces are specified by pairs consisting of quantity algebras and state spaces of the form $(\mathfrak{A}, \mathbf{S}(\mathfrak{H}))$, belonging to a single category. Likewise,
} 


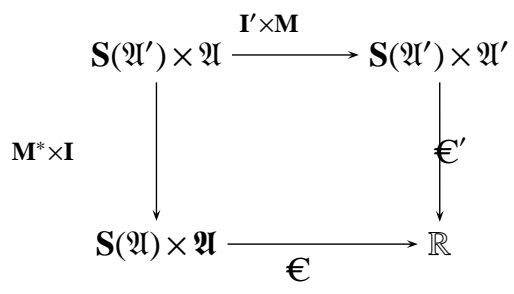

that serves perfectly well as a general description of the quantity-state duality we are using in this paper. In the diagram above, the evaluation map $€: \mathbf{S}(\mathfrak{A}) \times \mathfrak{A} \longrightarrow \mathbb{R}$, defined by the standard functional evaluation $€(\mathcal{S}, Q)={ }_{d f} \mathcal{S}(Q)$ for all states $\mathcal{S}$ and quantities $Q$, occupies a pivotal position in treating states and quantities as counterpart notions. Here 'commutativity' means that in the foregoing Chu space transformation diagram the two possible ways of composing maps along the arrows give the same result. That is to say, the adjointness condition

$$
\left[€^{\prime} \circ\left(\mathbf{I}^{\prime} \times \mathbf{M}\right)\right](\mathcal{S}, Q)=\left[€ \circ\left(\mathbf{M}^{*} \times \mathbf{I}\right)\right](\mathcal{S}, Q)
$$

holds for all states $\mathcal{S}$ in $\mathbf{S}\left(\mathfrak{H}^{\prime}\right)$ and for all quantities $Q$ in $\mathfrak{A}$. Upon applying the evaluation maps $€$ and $€^{\prime}$ to their arguments, the foregoing adjointness condition reduces to the equality $\mathcal{S}(\mathbf{M}(Q))=\left[\mathbf{M}^{*}(\mathcal{S})\right](Q)$, showing how the quantitychannel $\mathbf{M}$ and the state-channel $\mathbf{M}^{*}$ are related.

We have not yet considered how observers are to fit into the algebraic framework just outlined. ${ }^{16}$ Although we intend to study the structure of measurement operations in a strictly observer-independent manner, it must be recognized that measurement is more than just an exclusively physical process. A human observer's conscious mind wittinesses and receives information from the measuring instrument's dial through the eyes (or other organs) by reading (detecting) the finite-resolution values of the instrument's pointer quantity. Of course, there is nothing sacrosanct about this, since any good mechanical robot can accomplish practically this much. But there is more. We also have to consider how human observers interpret various measurement operations and how their subjective sensations of instrument dials result in beliefs about the measurand's values. And last but not least, in view of their powerful sense for effective idealization, human observers are amazing formal model builders with special abilities to recognize "interesting" dynamical behaviors. In conformity with our commitment to algebraic approaches to measurement, in this paper we shall not be concerned with the intriguing structure of mental states and the associated perceptual processes charactering pointer readings.

Since the physical content of a target system's algebraic model is largely contained in the quantity algebra associated with the system, we must first review some important facts

maps are also given by pairs, consisting of quantity- and state-channels. For more details, see [10].

${ }^{16}$ Signal processing theory of measurement (STM) treats the notion of observer systems-theoretically as a generalized measuring instrument that evaluates the difference between the target system's controlled input and measured output signals. about algebras and state spaces thereon. Another recurrent theme we shall take up later is the representation of measurement and other operations by suitable channels between quantity algebras.

\section{Algebraic FRAMEWORKS FOR MEASUREMENT}

In this section we present a universal algebraic paradigm, in which various models of measurement can be formulated in a unified manner. Although the basic framework is easy to describe, unfortunately, the conceptual building blocks require a number of definitions.

\subsection{Quantity algebras}

Quantity algebras generalize the familiar elementary operations on numbers. Specifically, a quantity algebra is a set $\mathfrak{A}$ with two basic binary operations on abstractly conceived quantities, namely the addition operation, written $Q+Q^{\prime}$ for two elements $Q$ and $Q^{\prime}$ in $\mathfrak{A}$, the multiplication operation symbolized $Q \bullet Q^{\prime}$, and the respective associated zero $\mathbf{0}$ and unit $\mathbf{1}$ elements of $\mathfrak{\mathfrak { A }}$. It is also important to include a scalar multiplication for scale change, denoted $c \cdot Q$ for any "scalar" $c$ in the field of reals $\mathbb{R}$ or complex numbers $\mathbb{C}{ }^{17}$ These operations satisfy certain axioms similar to those reserved for rings and modules. It is important to bear in mind that a quantity algebra does not say what quantities really are, only how they behave, relative to the algebra's operations. Algebras give specifications but do not determine implementation. Quantity algebras are also furnished with powerful topological or norm structures that are used in defining the limits of converging sequences of quantities, approximations, and the all-important continuity property of maps between algebras.

Clearly, length, mass, energy and all the other so-called extensive quantities can be combined additively. Furthermore, length can be multiplied with itself to get an area, volume, or an arbitrary high-dimensional hyper-volume. As an application of Occam's razor, in any quantity algebra we allow all kinds of quantities expressed by polynomials that may lack physical significance. ${ }^{18}$ It must be emphasized that a given quantity may be introduced as a basic (primitive) or as a derived (defined) notion. For example, the concept of energy remains unspecified in mainstream algebraic models of classical mechanical systems without the introduction of length, mass

\footnotetext{
${ }^{17}$ In the ambience of complex scalars, quantity algebras are usually equipped with a special complex conjugation-based unary operation $Q^{\dagger}$, called involution, forming $\dagger$-algebras or $*$-algebras, needed in modeling the behavior of quantum systems.

${ }^{18}$ In opting for the conceptually simplest and most economic algebraic model, it is standard to admit certain mathematical elements in the model that are devoid of physical meaning, as long as the model also possesses a rich supply of empirically significant elements in its applicable subdomain. For example, the classical pendulum equation physicists regularly use admits infinitely many solutions that are specified by physically meaningless superluminal velocities. In the case of quantity algebras, there is a choice between complex partial algebras, riddled with ad hoc constraints that exclude all empirically insignificant quantities and traditional normed algebras that are likely to include quantities ruled out by applications.
} 
and time quantities. In contrast, in algebraic models of quantum systems the notion of energy is usually specified independently of the mass quantity. Of course, it is always possible to introduce the energy quantity as basic. Upon amalgamating the structure of quantity algebras and the definitional or lawlike relations between quantities, we find that the description of the target system's behavior cannot be said to be complete without additional (usually equational) constraints, provided by special maps (operators) between quantity algebras. We shall give further details later.

The so-called intensive quantities that are often defined (e.g., via differentiation) in terms of pairs of extensive quantities (including density, pressure, and electric, chemical and gravitational potentials) tend to arise as parameters of the target system's states and extensive quantities, and often form a convex subspace of the underlying quantity algebra or that of the algebra's conjugate or (pre)dual space.

Quantity algebras are blessed with positive quantities, symbolized by the inequality $Q \geq \mathbf{0}$ and having the form $Q=$ $Q^{\prime} \cdot Q^{\prime}$ for some quantity $Q^{\prime}$ in $\mathfrak{B}$. It is elementary to verify that positive quantities form a convex cone and thus induce a natural linear partial ordering, denoted $Q \geq Q^{\prime}$ and defined by $Q-Q^{\prime} \geq \mathbf{0}$. To increase the applicability of quantity algebras, we are striving for additional operations on quantities. Curiously, each positive quantity $Q$ of $\mathfrak{B}$ has a square root, symbolized $\sqrt{Q}$ and is given by the unique quantity $Q^{\prime}$ such that the equality $Q=Q^{\prime} \cdot Q^{\prime}$ holds. Obviously, positive quantities have positive numerical values. Of primary importance for us is the notion of an invertible quantity. A quantity $Q$ in a given algebra $\mathfrak{A}$ is said to be invertible provided that the equalities $Q \cdot Q^{\prime}=\mathbf{1}=Q^{\prime} \cdot Q$ hold for a unique quantity $Q^{\prime}$ in $\mathfrak{A}$, symbolized $Q^{-1}$ and called the inverse of $Q$. For future needs, we denote the multiplicative group of invertible elements of quantity algebra $\mathfrak{A}$ by $\mathfrak{A}^{-1}$. We can now define the spectrum (i.e., the set of possible values) of quantity $Q$ strictly algebraically as follows:

$$
\operatorname{Spec}(Q)=_{d f}\left\{c \in \mathbb{R} \mid c \cdot \mathbf{1}-Q \notin \mathfrak{A}^{-1}\right\}
$$

We remark in passing that the spectra (i.e., value spaces) of various derived quantities are obtained by the following algebraic specifications: $\operatorname{Spec}(\mathbf{1})=\{1\}, \operatorname{Spec}(\sqrt{Q})=\{\sqrt{c} \mid c \in$ $\operatorname{Spec}(Q)\}, \operatorname{Spec}(Q \bullet Q)=\left\{c^{2} \mid c \in \operatorname{Spec}(Q)\right\}$, and $\operatorname{Spec}\left(Q^{-1}\right)=$ $\left\{c^{-1} \mid c \in \operatorname{Spec}(Q)\right\}$, if $Q^{-1} \in \mathfrak{A}^{-1}$. In the case of exponential quantities (defined by the familiar convergent series) we have $\operatorname{Spec}\left(\mathbf{e}^{t Q}\right)=\left\{\mathbf{e}^{t c} \mid c \in \operatorname{Spec}(Q)\right\}$, and likewise for $\log Q, \sin (t Q)$ and the other elementary functions.

\subsection{Construction and design of quantity algebras}

How should quantity algebras be constructed? In practice, the choice of a quantity algebra for the target system involves a well-informed judgment and the algebra's correctness and that of accompanying physical laws are experimentally checked by comparing the system's measurement results with the predictions generated by the algebra and laws. Any significant discrepancy between algebraic model-generated predictions and measurement results renders the model inadequate and prompts its replacement by a more detailed or altogether different algebra. The choice of quantities and the size (or degrees of freedom) of the generated algebra depend on the desired level of description (from coarse-grained to fine-grained), and on the stipulated predictive accuracy and resolution of the target system's behavior. Stated differently, the quantities for the target system should be selected in such a way that from the knowledge of their values and laws the experimenter can reliably determine the system's behavior to within an antecedently specified degree of accuracy. Unfortunately, model-based computations and instrument-based measurements cannot be accomplished without some form of approximation - dictated not only by the required accuracy, but also by mathematical and instrumental tractability. Although it may seem correct to think that the higher the desired accuracy, the larger the modeling algebra of quantities (carrying more information), this relationship fails epistemically, when tractability becomes an issue (e.g., high-accuracy models of classical macroscopic systems based on noncommutative quantum system algebras are hopelessly complex and intractable).

Importantly, even if the initial choice of a particular quantity algebra may be subjective and some values of some quantities are not determinable by measurement, the validity (or invalidity) of the accepted algebraic model is an objective property and so is the system's state. Needless to add, the measured value of a measurand (read off from the calibrated instrument's dial) is the measuring instrument's property.

To capture quantity entanglements associated with two (or more) systems $S$ and $\mathbf{S}^{\prime}$, the representing algebras $\mathfrak{2}_{\mathbf{S}}$ and $\mathfrak{P}_{\boldsymbol{S}^{\prime}}$ are combined into their tensor product algebra $\mathfrak{\Re}_{\mathbf{S}} \otimes \mathfrak{A}_{\mathbf{S}^{\prime}}$, generated by elementary tensor quantities of the form $Q \otimes Q^{\prime}$ with $Q$ in $\mathfrak{A}_{\mathrm{S}}$ and $Q^{\prime}$ in $\mathfrak{\Re}_{\mathrm{S}^{\prime}}$. Since tensor products tend to lack canonical projection and diagonal maps, they are significantly more general than the usual direct (Cartesian) products. Tensor product algebras are needed in modeling physical interactions between coupled systems that allow entangled states. As with many other algebraic constructions, tensor product algebras are unique only up to an algebra isomorphism relation between them. We denote the isomorphism relation between quantity algebras $\mathfrak{A}$ and $\mathfrak{P}^{\prime}$ by $\mathfrak{A} \cong \mathfrak{H}^{\prime}$.

\subsection{Quantity-channels}

It is high time we had the precise definition of a quantitychannel from the duality-based algebraic point of view we have been advocating. A map of the form

$$
\mathfrak{A} \stackrel{\mathbf{M}}{\longrightarrow} \mathfrak{A}^{\prime}
$$

from quantity algebra $\mathfrak{A}$ to quantity algebra $\mathfrak{P I}^{\prime}$ is called a quantity-channel provided that the following conditions are satisfied for all quantities $Q, Q^{\prime}$ and sequences $Q_{1}, Q_{2}, \cdots$ in $\mathfrak{I}$, and for all scalars $a, b \in \mathbb{R}$ :

(i) Linearity: $\mathbf{M}\left(a Q+b Q^{\prime}\right)=a \mathbf{M}(Q)+b \mathbf{M}\left(Q^{\prime}\right)$. 
(ii) Unity: $\mathbf{M}(\mathbf{1})=\mathbf{1}$.

(iii) Positivity: $Q \geq \mathbf{0} \Longrightarrow \mathbf{M}(Q) \geq \mathbf{0}$.

(iv) Weak continuity: If $\operatorname{wlim}_{n \rightarrow \infty} Q_{n}=$ $Q$, then wlim $_{n \rightarrow \infty} \mathbf{M}\left(Q_{n}\right)=\mathbf{M}(Q)$, where the so-called weak limit in the algebra $\mathfrak{A}$ is defined by

$$
\left.\operatorname{wlim}_{n \rightarrow \infty} Q_{n}=Q \Longleftrightarrow \lim _{n \rightarrow \infty} \mathcal{S}\left(Q_{n}-Q\right)\right)=0
$$

for all states $\mathbf{S}$ in state space $\mathbf{S}(\mathfrak{H})$. We shall encounter the formal definition of the notion of state shortly.

In typical applications to measurement, it is helpful to think of quantity-channels as mappings that send instrument quantities to various estimates or approximations of measurands. Clearly, some quantity-channels are better than others in this respect.

\subsection{Determining the values of quantities}

We now move to the next conceptual issue of how the values of quantities at any particular time are specified (deterministically or probabilistically) by the target system's internal conditions at that time, referred to as the system's physical state. As far as the nature of physical states is concerned, it can be as simple as a particle's position coordinate and momentum value or as complex as the "statistical mode of being" of a material body made up of an enormous number (e.g., greater than $10^{20}$ ) of interacting particles, investigated in statistical mechanics. In this connection it is revealing to look at the state as a snapshot of the way the system is at each moment of time that gives rise to a snapshot of the current values of quantities. Bear in mind that these snapshots tend to have statistical content, requiring a probabilistic encoding in terms of suitable probability measures. We know that a single reading of a pointer is subject to several kinds of errors that cannot be eliminated. In realistic settings, repeated measurements of measurand $Q$ under identical or exchangeable conditions result in a probability distribution (approximated by a histogram) on $\operatorname{Spec}(Q)$ rather than in a sharp value, concentrated at one point in the value space. Even if the target system's state is prepared in the laboratory in a maximally precise way, due to random noises the result of measurement of $Q$ will generally not be a single point, but a nontrivial probability distribution on $\operatorname{Spec}(Q)$.

To obtain a sufficiently general framework for representing the variety of physical states and quantity values discussed above, we shall suppose that a target system is always in some kind of a statistical state (including deterministic limit-case situations) that is responsible (probabilistically or deterministically) for the extant values of quantities.

The question now arises as to how did the system get to be the way it is now? Empiricists have an answer and it is the following: The system has been prepared to be that way by its past interactions with other systems. From this point of view the system's state can be thought of as an equivalence class of potential preparation procedures applied to the system which gives the same probability distribution for all measurement outcomes. In a dual setting, a quantity may be viewed as an equivalence class of possible measurement procedures which gives the same probability distribution for all preparations. This is all very suggestive, but we find the opposing objectivist approach to classical measurement not only ontologically more meager but also more effective. Specifically, earlier we have assumed that the system's quantities possess their values independently of whether or not they are measured. From this perspective, the system is in its current state, irrespective of whether or not the experimenter knows anything about the system's preparatory history.

\subsection{State spaces and state-channels}

In accord with the accepted algebraic paradigm, we next introduce special spaces, called state spaces, the elements of which are algebraic states (or statistical states as they are also commonly known), representing the possible physical modes of being of systems at various moments of time. It is evident that there are three basic options in the mathematical formulation of algebraic states. One alternative is to declare algebraic states as basic (undefined) notions, paralleling the independently introduced concept of quantity. Another viewpoint is to start with algebraic states and treat quantities as state functions of some kind. The view we shall adopt in this paper is to define algebraic states with reference to the target system's quantity algebra. Proceeding in this manner means that quantities are viewed as primary, while algebraic states are in some sense complementary, encoding the decisive dual (statistical) structure of quantities.

The upshot of all of this is that we may introduce algebraic states in terms of quantities or view quantities as functions on states. For us, it is the power of the algebraic approach that motivates our preferred way of defining states as special functions on quantity algebras. So what is the conceptual significance of algebraic states in measurement theory? Unquestionably, the most significant import of algebraic states in modeling measurement operations is a structural enrichment needed for (i) the statistical description of quantities in terms of probability distributions of their possible values and the accompanying statistical indicators, such as averages and variance, (ii) state-channels, representing information transfers from measurands to their pointer quantities, and for (iii) deepening the interpretation of measurement models. We address this topic in more technical detail below.

We have already indicated that with quantity algebras $\mathfrak{A}$ and quantity-channels in the picture, in the dual setting of the algebraic approach to measurement the investigator associates with each system $S$ in the given domain of reality a convex space $\mathbf{S}\left(\mathfrak{I}_{\mathbf{S}}\right)$ of algebraic states on $\mathfrak{A}$, belonging or extendable to the algebra's dual $\mathfrak{A}^{*}$, i.e., we have $\mathbf{S}(\mathfrak{A}) \subset \mathfrak{A}^{*}$.

By a faithful normal algebraic state, henceforth simply state, we mean a real-valued linear function of the form 
$\mathcal{S}: \mathfrak{A} \longrightarrow \mathbb{R}$ that satisfies the following conditions for all quantities $Q$ and sequences $Q_{1}, Q_{2}, \cdots$ in $\mathfrak{A}$ :

(i) Unity: $\mathcal{S}(\mathbf{1})=1$.

(ii) Positivity: $Q \geq \mathbf{0} \longrightarrow \mathcal{S}(Q) \geq 0$.

(iii) Continuity: $\lim _{n \rightarrow \infty}\left\|Q_{n}-Q\right\|=0 \Longrightarrow \lim _{n \rightarrow \infty} \mid \mathcal{S}\left(Q_{n}\right)-$ $\mathcal{S}(Q) \mid=0$

(iv) Faithfulness: If $Q \geq \mathbf{0}$, then $\mathcal{S}(Q)=0 \Longrightarrow Q=\mathbf{0}$.

Since in classical measurement we encounter mostly faithful and normal algebraic states, and because these natural states are required by quantity-state duality, in what follows we shall always work with faithful normal states. Accordingly, unless the contrary is explicitly stated, the state space $\mathbf{S}(\mathfrak{A})$ will always be specified by the set of all faithful normal algebraic states on quantity algebra $\mathfrak{A}$. Here, the numerical value $\mathcal{S}(Q)$ is interpreted as the average (or expectation) of quantity $Q$, given that the target system is in a physical state represented by $\mathcal{S}$.

Turning to the structure of state spaces, perhaps the most important one is their closure under the familiar mixing operation that is widely used in probability calculus. Specifically, if states $\mathcal{S}, \mathcal{S}^{\prime}$ are in $\mathbf{S}(\mathfrak{H})$, then their convex mixture defined by $c \cdot \mathcal{S}+(1-c) \cdot \mathcal{S}^{\prime}$ (with $0 \leq c \leq 1$ is also in $\mathbf{S}(\mathfrak{A})$. Thus, states form a convex space. Each state space comes with its lattice of faces and other related structure, but we will not pursue the details. A state is said to be pure (extreme or deterministic) provided that it is not a proper mixure of any pair of other states. Formally, a state $\mathcal{S}$ is pure just in case for any pair $\mathcal{S}_{1}$ and $\mathcal{S}_{2}$ of states and a scalar $0<c<1$ the following conditional

$$
\mathcal{S}=c \cdot \mathcal{S}_{1}+(1-c) \cdot \mathcal{S}_{2} \Longrightarrow \mathcal{S}=\mathcal{S}_{1}=\mathcal{S}_{2}
$$

holds. Since pure states play a crucial role in deterministic measurement, for future needs, we denote the subset of pure states by $\mathbf{S}_{e x}(\mathfrak{A})$. Importantly, for any pure state $\mathcal{S}$ the average $\mathcal{S}(Q)$ is actually the extant value of quantity $Q$.

Next, we need to recall some facts from information theory about state-channels, arising (by duality) from quantitychannels. We begin with the pertinent formal definition. By a state-channel we mean a map from an input state space $\mathbf{S}\left(\mathfrak{A}^{\prime}\right)$ to an output state space $\mathbf{S}(\mathfrak{A})$ of the form

$$
\mathbf{S}\left(\mathfrak{I}^{\prime}\right) \stackrel{\mathbf{M}^{*}}{\longrightarrow} \mathbf{S}(\mathfrak{H}),
$$

defined by the composition $\mathbf{M}^{*}(\mathcal{S}){ }_{d f} \mathcal{S} \circ \mathbf{M}$ for all states $\mathcal{S}$ in $\mathbf{S}(\mathfrak{A})$, where $\mathbf{M}$ is a previously specified quantity-channel. It is important to appreciate a converse definition, stating that the quantity channel $\mathbf{M}$ can be specified in terms of a given state-channel $\mathbf{M}^{*}$ as follows: $\mathbf{M}(Q)=_{d f} Q^{\prime}$, where $\mathcal{S}\left(Q^{\prime}\right)=$ $\left.\mathbf{M}^{*}(\mathcal{S})\right)(Q)$ for all $\mathcal{S}$.

Returning to the general case, a state-channel is any map of the form $\mathbf{M}^{*}: \mathbf{S}\left(\mathfrak{H}^{\prime}\right) \longrightarrow \mathbf{S}(\mathfrak{H})$ that satisfies the following conditions: (i) Positivity: $\mathbf{M}^{*}$ sends input states to output states.

(ii) Convexity: $\mathbf{M}^{*}$ has the so-called affine property

$$
\mathbf{M}^{*}\left(c \cdot \mathcal{S}+(1-c) \cdot \mathcal{S}^{\prime}\right)=c \cdot \mathbf{M}^{*}(\mathcal{S})+(1-c) \cdot \mathbf{M}^{*}\left(\mathcal{S}^{\prime}\right)
$$

for all states $\mathcal{S}$ and $\mathcal{S}^{\prime}$ in $\mathbf{S}\left(\mathfrak{A}^{\prime}\right)$, and for all scalars $0 \leq$ $c \leq 1$.

The foregoing affine (convexity) property captures the familiar randomization or stochastic equivalence principle: Any target system in state $\mathcal{S}$ with probability $c$ and in state $\mathcal{S}^{\prime}$ with probability $1-c$ cannot be empirically distinguished from the system in state $c \cdot \mathcal{S}+(1-c) \cdot \mathcal{S}^{\prime}$.

There are special conditions investigators regularly impose on state-channels in various contexts. We mention just three of them to standardize our terminology:

(i) A state-channel $\mathbf{M}^{*}$ of the form above is called pure just in case the inclusion $\mathbf{M}^{*}\left(\mathbf{S}_{e x}(\mathfrak{H})\right) \subseteq \mathbf{S}_{e x}\left(\mathfrak{I}^{\prime}\right)$ holds. Intuitively, pure state-channels map pure states to pure states. A channel $\mathbf{M}^{*}$ is pure if and only if $\left[\mathbf{M}^{*}(\mathcal{S})\right](P)=1$ or 0 for all two-valued (projection) quantities $P$ and pure states $\mathcal{S}$.

(ii) The state-channel $\mathbf{M}^{*}$ referred to above is called orthogonal provided that it preserves the orthogonality of states, i.e. we have $\mathcal{S} \perp \mathcal{S}^{\prime} \Longrightarrow \mathbf{M}^{*}(\mathcal{S}) \perp \mathbf{M}^{*}\left(\mathcal{S}^{\prime}\right)$, where the orthogonality relation $\mathcal{S} \perp \mathcal{S}^{\prime}$ is satisfied just in case the states $\mathcal{S}$ and $\mathcal{S}^{\prime}$ belong to a disjoint pair of faces of the state space $\mathbf{S}\left(\mathfrak{I}^{\prime}\right)$.

(iii) A state-channel $\mathbf{M}^{*}$ is called deterministic if and only if it is one-to-one (bijective) and orthogonal. Deterministic state-channels send pure states to pure states in a one-toone fashion.

In applications involving static measurement operations, state-channels take as an argument the target system's extant state and send it to the measuring instrument's current state. The physical significance of state-channels is the following: Channels transmit input information carried by the system's extant state to the instrument, where it arrives in the form of a (generally) reduced or corrupted information, encoded by the instrument's state.

In sum, the central point of algebraic theories of measurement we will explore via examples can now be stated very simply as follows:

1. To each system $S$ belonging to a partially ordered monoid of systems $\langle\mathbf{S}, \mathrm{O},+, \preccurlyeq\rangle$ we associate a quantity algebra $\mathfrak{A}_{S}{ }^{19}$ in such a way that the following conditions are satisfied for all systems $\mathbf{S}$ and $\mathbf{S}^{\prime}$ in $\mathbf{S}$ :

\footnotetext{
${ }^{19}$ The associated quantity algebra $\mathfrak{P}_{\mathrm{S}}$ together with its dynamical structure, discussed in [3], is intended to capture the target system's basic behavioral features of interest. Additional structures are needed to describe the system's perturbation and interactions with other systems.
} 
(a) $\mathfrak{P}_{S_{+} S^{\prime}} \cong \mathfrak{P}_{S} \otimes \mathfrak{P}_{S}^{\prime}$.

(b) $\mathfrak{I}_{\mathrm{O}} \cong \mathbb{R}$.

(c) If $\mathrm{S} \leqslant \mathrm{S}^{\prime}$, then there exists an embedding algebra homomorphism $\mathbf{J}: \mathfrak{A}_{\mathrm{S}} \longrightarrow \mathfrak{A}_{\mathrm{S}}^{\prime}$.

2. To each elementary measurement operation $\mathscr{M}: \mathrm{S} \rightarrow \mathrm{M}$ we associate a quantity-channel $\mathbf{M}: \mathfrak{A}_{\mathbf{M}} \longrightarrow \mathfrak{A}_{\mathrm{S}}$ that represents the inferential transfer of data from the measuring instrument's quantity algebra to the system's algebra.

In parallel with the above, there is also a dual association of state space and state-channels. A quantity-channel $\mathbf{M}$ and its associated state-channel $\mathbf{M}^{*}$ are two complementary representations of the same measurement operation.

In the universe of algebraic models we adopt there are three major kinds of quantity algebras of measurement-theoretic interest, earmarked specifically for smooth, continuous and measurable quantities (measurable in the sense of classical measure theory), which have been given appropriate names. One further feature of quantities should be mentioned: the spectrum (value space) of each quantity in an algebra can be a subset of the field $\mathbb{R}$ of real numbers or that of the field $\mathbb{C}$ of complex numbers. Although complex numbers greatly simplify various technical problems and support the correct level of generality, known to be essential for the construction of quantity algebras of quantum systems and quantum fields, in this paper we find it convenient to work over the base field of reals. Since smooth (i.e., infinitely differentiable) quantities are continuous and continuous quantities are measurable, we shall discuss only the algebras of measurable quantities.

\section{Von Neumann algebras of MEASURAble QUANTITIES}

Next to Banach algebras of continuous quantities, the simplest and most widely used class of quantity algebras is given by real and complex von Neumann algebras of measurable quantities. Unfortunately, von Neumann algebras can be characterized in many ways, some abstract and others concrete, based on Hilbert-space operator algebras, but none of them seems sufficiently intuitive. Importantly, von Neumann algebras are Banach spaces ${ }^{20}$, just like Banach algebras. However, the main difference between Banach algebras and von Neumann algebras is a large supply of idempotent (two-valued) quantities in the latter, satisfying the idempotence law $Q \bullet Q=$ $Q$, also known as projection quantities. Projection quantities will play a fundamental role in much of what follows. Specifically, each quantity in a von Neumann algebra is canonically representable by a weighted sum or integral of projection quantities. We shall give some specifics below. Von Neumann algebras are also important for their intimate relationship to their conjugate (predual) Banach spaces. As we shall

\footnotetext{
${ }^{20}$ Recall that a Banach space is a normed vector space that is complete with respect to the metric induced by the norm.
}

see next, von Neumann algebras (as Banach spaces) are always duals of something, namely, they are duals of Banach spaces.

By definition, a normed algebra $\mathfrak{A}$ is said to be a von $\mathrm{Neu}$ mann algebra provided that it is the dual Banach space of another uniquely given Banach space, symbolized $\mathfrak{A}_{*}$ and called the predual or conjugate of algebra $\mathfrak{A}$, so that the algebra isomorphism $\mathfrak{A} \cong\left(\mathfrak{A}_{*}\right)^{*}$ holds. $^{21}$

\subsection{Measure-theoretic representation of commutative von Neumann algebras}

It is well known that every probability space $\langle\mathscr{X}, \mathcal{B}, \mathbf{P}\rangle$ determines a unique real unital commutative von Neumann algebra - traditionally denoted by the Lebesque space symbolism $\mathfrak{L}_{\infty}(\mathscr{X}, \mathcal{B}, \mathbf{P})$ - of all essentially bounded real-valued random variables of the form $f: \mathscr{X} \longrightarrow \mathbb{R}$, where this time two random variables $f$ and $f^{\prime}$ on $\mathscr{X}$ are identified, whenever they are equal $\mathbf{P}$-almost everywhere, meaning the induced equivalence relation $f \sim f^{\prime}$ holds. As anticipated, the equivalence is defined by $f \sim f^{\prime}$ if and only if $\mathbf{P}\left(\llbracket f=f^{\prime} \rrbracket\right)=1$. Here and in what follows we use the notation $\llbracket f=f^{\prime} \rrbracket$ for the measurable set $\left\{x \mid f(x)=f^{\prime}(x)\right\}$ in $\mathcal{B}^{22}$

Essential boundedness in $\boldsymbol{L}_{\infty}(\mathscr{X}, \mathcal{B}, \mathbf{P})$ means the finiteness of the canonical essential supremum norm $\|f\|_{\infty}$, defined by the supremum formula

$$
\|f\|_{\infty}=_{d f} \sup \{c>0 \mid \mathbf{P}(\{x \in \mathscr{X}|| f(x) \mid>c\})>0\} .
$$

The definition says that the absolute value of each measurable quantity $f$ is bounded by a positive constant $\mathbf{P}$-almost everywhere. Boundedness allows to work with arbitrarily long multiplications of quantities. As usual in analysis, the algebra's operations are defined pointwise by setting $\left[f+f^{\prime}\right](x)=_{d f} f(x)+f^{\prime}(x)$ and $\left[f \bullet f^{\prime}\right](x)=_{d f} f(x) \cdot f^{\prime}(x)$

\footnotetext{
${ }^{21}$ Banach algebras do not have a predual Banach space and they need not have any projection quantities, other than $\mathbf{0}$ and $\mathbf{1}$. From a category-theoretic perspective, the dual construction leading from space $\mathfrak{A}$ to its dual space $\mathfrak{A}^{*}$ (defined by the space of bounded linear functionals on $\mathfrak{A}$ ), is a contravariant functor from the category of Banach spaces to itself. Along similar lines, the predual construction is given by a contravariant functor from the category of von Neumann algebras to the category of Banach spaces.

${ }^{22}$ Strictly speaking, the elements of $\mathbf{Q}_{\infty}(\mathscr{X}, \mathcal{B}, \mathbf{P})$ are not functions, but equivalence classes of functions that happen to agree $\mathbf{P}$-almost everywhere, i.e., everywhere except on the physically unimportant subsets of $\mathbf{P}$-measure zero. For this reason, the spectrum of a measurable quantity $f$ is defined in terms of its essential range

$$
\operatorname{Spec}(f)={ }_{d f}\{c \mid \forall \varepsilon>0[\mathbf{P}(\{x \in \mathscr{X}|| f(x)-c \mid<\varepsilon\})>0]\} .
$$

In words, a numerical value $c$ belongs to the essential range of $f$ (and hence to $f$ 's spectrum) if and only if every neighborhood of $c$ has a strictly positive probability measure. The idea of equivalence classes of quantities can be circumvented by viewing quantities as measurable functions of the form $f: \mathscr{X}_{0} \longrightarrow \mathbb{R}$, defined on a P-conegligible subset (obtained from $\mathscr{X}$ by subtracting all sets of $\mathbf{P}$-measure zero)
}

$$
\mathscr{X}_{0}=\left\{x \in \mathscr{X} \mid \forall_{B \in \mathcal{B}}[x \in B \Longrightarrow \mathbf{P}(B)>0]\right\} .
$$


for all $x$ in $\mathscr{X}$. We also need the usual scalar multiplication, defined by $[c \cdot f](x)=_{d f} c \cdot f(x)$. The essential supremum norm turns the algebra $\mathfrak{L}_{\infty}(\mathscr{X}, \mathcal{B}, \mathbf{P})$ into a Banach space with its predual (conjugate) Banach space $\mathfrak{L}_{1}(\mathscr{X}, \mathcal{B}, \mathbf{P})$ of absolutely integrable functions (containing all density functions) with a finite norm $\|f\|_{1}$, defined by the integral $\|f\|_{1}=_{d f} \int_{\mathscr{X}}|f(x)| \mathbf{P}(\mathrm{d} x)<\infty$. The following important predual and dual algebra-isomorphisms hold: $\mathfrak{L}_{\infty}(\mathscr{X}, \mathcal{B}, \mathbf{P})_{*} \cong$ $\mathfrak{L}_{1}(\mathscr{X}, \mathcal{B}, \mathbf{P})$ and $\mathfrak{I}_{1}(\mathscr{X}, \mathcal{B}, \mathbf{P})^{*} \cong \mathfrak{L}_{\infty}(\mathscr{X}, \mathcal{B}, \mathbf{P})$.

It is well known in operator theory that any abstractly given unital commutative von Neumann algebra ${ }^{23}$ is isomorphic to a concrete von Neumann algebra $\mathfrak{Q}_{\infty}(\mathscr{X}, \mathcal{B}, \mathbf{P})$ for some probability space $\langle\mathscr{X}, \mathcal{B}, \mathbf{P}\rangle$ with its to-within-isomorphism unique predual Banach space $\mathfrak{Q}_{1}(\mathscr{X}, \mathcal{B}, \mathbf{P})$. Remarkably, the algebra $\mathbf{R}_{\infty}(\mathscr{X}, \mathcal{B}, \mathbf{P})$ depends only on the sigma ideal of $\mathbf{P}$-null sets and not on the specific choice of the measure $\mathbf{P}$. Therefore, for notational convenience we introduce the abbreviation $\mathfrak{Q}_{\infty}(\mathscr{X})$ for $\mathfrak{Q}_{\infty}(\mathscr{X}, \mathcal{B}, \mathbf{P})$.

Because the objective of classical measurement is to identify the extant value of the target system's selected measurand, it is important to understand which other quantities (determined by the measurand) will also acquire known values upon completing the measurand's measurement. Put another way, suppose the experimenter measures measurand $Q$ and obtains its value, say, $c \in \operatorname{Spec}(Q)$ or the best estimate thereof. Evidently, the experimenter does not have to perform another measurement to know the value, say, of the squared measurand $Q^{2}=Q \cdot Q$, because (s)he already knows that the value of $Q^{2}$ will be $c^{2}$ or its best estimate, obtained by easy (approximate) calculation. Needless to add, we can quickly generalize the foregoing idea of derived measurement to any 'scalechanging' real-valued measurable function $f$ on $\operatorname{Spec}(Q)$, giving the value $f(c)$ (or its approximation) of $f(Q)$. Simply, by measuring measurand $Q$ the experimenter automatically "measures" in a derived (computational) fashion all quantities of the form $f(Q)$.

\subsection{Measurable function calculus}

The so-called spectral mapping theorem allows us to say considerably more about how to move between the measurand's abstract von Neumann algebra and the associated concrete measurable function algebra. For the general case, let $Q$ be a measurand of interest in a von Neumann algebra $\mathfrak{A}$ and let $\mathfrak{A}(Q)$ be the von Neumann algebra generated (within $\mathfrak{A}$ ) by $Q$ and the algebra's unit $\mathbf{1}$. It is not hard to see that the resulting commutative von Neumann algebra $\mathfrak{A}(Q)$ (i.e., the smallest unital von Neumann subalgebra of $\mathfrak{A}$ containing measurand $Q$ and unit 1) is given by the norm closure of all polynomials in indeterminate $Q$. Simply, the generated algebra includes all quantities whose values can be obtained via derived measurement, based on $Q$. An important consequence of the spectral

\footnotetext{
${ }^{23}$ Noncommutative complex von Neumann algebras are reserved for quantum systems. Since our interest is in classical measurement, in this paper we shall focus only on commutative von Neumann algebras, satisfying the commutativity law $Q \cdot Q^{\prime}=Q^{\prime} \bullet Q$
}

mapping theorem which will be regularly used later in various measurement examples is the existence of a unique von Neumann algebra isomorphism

$$
\mathbf{L}_{\infty}(\operatorname{Spec}(Q)) \stackrel{\mathbf{I}_{Q}}{\longrightarrow} \mathfrak{A}(Q)
$$

between the algebra $\boldsymbol{\mathfrak { Q }}_{\infty}(\operatorname{Spec}(Q))$ of essentially bounded Borel measurable functions of the form $f: \operatorname{Spec}(Q) \rightarrow \mathbb{R}$ (where $\operatorname{Spec}(Q)$ is furnished with a unique probability measure $\mathbf{P}$ on the Boolean sigma-algebra of Borel subsets, modulo measure class equivalence, ${ }^{24}$, and the von Neumann algebra $\mathfrak{A}(Q)$ generated by the measurable quantity $Q$ and the unit $\mathbf{1}$ of $\mathfrak{A}$.

The isomorphism $\mathbf{I}_{Q}$ sends the canonical identity function $\mathfrak{I}: \operatorname{Spec}(Q) \rightarrow \mathbb{R}$ (defined by $\mathfrak{J}(x)=x$ for all $x$ in $\operatorname{Spec}(Q))$ directly to $Q=\mathbf{I}_{Q}(\mathfrak{J})$, it maps the constant function $k_{1}: \operatorname{Spec}(Q) \longrightarrow \mathbb{R}$ (having the same value 1 ) to the algebra's unit 1 , it assigns to the square function on $\operatorname{Spec}(Q)$ the quantity $Q^{2}$, and so forth. Here the main technical point is that the foregoing isomorphism ensures that we can locally manipulate the abstractly given quantities and representing measurable functions in the same way. For a detailed account of algebra representation results, the reader is referred to [12].

To mirror the algebraic and metrical properties of measurable functions in the generated quantity algebra $\mathfrak{A}(Q)$, it is customary to set $f(Q)={ }_{d f} \mathbf{I}_{Q}(f)$ to be the image of the measurable function $f: \operatorname{Spec}(Q) \longrightarrow \mathbb{R}$ under isomorphism $\mathbf{I}_{Q}{ }^{25}$ Although the foregoing notation does not seem natural at first sight, it does make good sense after showing that the following linear and structural conditions hold for all real-valued measurable functions $f, g$, and $f_{t}$ on $\operatorname{Spec}(Q)$ and scalars $c, t \in \mathbb{R}:$

(i) $[f+g](Q)=f(Q)+g(Q)$.

(ii) $[f \bullet g](Q)=f(Q) \bullet g(Q)$.

(iii) $[c \cdot f](Q)=c \cdot f(Q)$.

(iv) If $f \leq g$, then $f(Q) \leq g(Q)$.

(v) If $f_{t}(x)=\mathbf{e}^{-t x}$, then $f_{t}(Q)=\mathbf{e}^{-t Q}$.

(vi) If $\lim _{n \rightarrow \infty} f_{n}=f$, then $\lim _{n \rightarrow \infty} f_{n}(Q)=f(Q)$.

(vii) $[h \circ f](Q)=h(f(Q))$, where $h \in \mathbf{Q}_{\infty}(f(\operatorname{Spec}(Q)))$.

(viii) $\|f(Q)\|=\sup \{|f(c)| \mid c \in \operatorname{Spec}(Q)\}$.

(ix) $\operatorname{Spec}(f(Q))=f(\operatorname{Spec}(Q))=\{f(c) \mid c \in \operatorname{Spec}(Q)\}$.

Basically, as alluded to above, there are two mathematically equivalent ways to reason about a measurand and

\footnotetext{
${ }^{24}$ As was mentioned above, the probability measure $\mathbf{P}$ is needed only for ensuring appropriately many null sets.

${ }^{25}$ Because $f(Q)$ denotes the quantity that corresponds to the measurable function $f$ under the algebra isomorphism, mathematicians working in spectral analysis often refer to this type of correspondence as the measurable function calculus.
} 
its derived quantities, (i) algebraically, and (ii) measuretheoretically. In the algebraic approach, the measurand's spectrum $\operatorname{Spec}(Q)$ automatically specifies the spectrum of any derived quantity, having the polynomial form $c_{0} \cdot \mathbf{1}+c_{1}$. $Q+c_{2} \cdot Q^{2}+\cdots+c_{n} \cdot Q^{n}$ or a limit thereof with respect to the norm-induced topology. From a measure-theoretic perspective, all calculations of values of derived quantities are performed on the corresponding measurable functions of the form $f: \operatorname{Spec}(Q) \longrightarrow \mathbb{R}$. Since the measure-theoretic approach is equivalent to the algebraic method, why bother with the algebras of abstract quantities at all? Because the algebraic approach is particularly well-suited to modeling not only classical measurement, but also measurement procedures arising in quantum mechanics and quantum statistical mechanics, where (due to noncommutativity requirements) concrete measuretheoretic representations are not available. In particular, algebraic models provide (at the right level of generality and simplicity) a unified mathematical framework for the study of all known types of measurement operations, including but not limited to classical and quantum, static and dynamical, deterministic and probabilistic, direct and indirect, discrete-time and continuous-time, joint, repeatable, parallel and sequential, biased and unbiased, invasive and noninvasive, demolition or nondemolition, and model-based measurements.

Since for any measurable set $B \in \mathcal{B}$, its characteristic function $\mathbf{1}_{B}$ is automatically a (2-valued, idempotent) projection quantity in $\mathfrak{2}_{\infty}(\mathscr{X})$, the set of projection quantities forms a Boolean sigma algebra, mirroring the underlying Boolean algebra of propositions. ${ }^{26}$ We have thus established that all externally given (data) propositions are automatically internalized in a von Neumann algebra in terms of projection quantities. $^{27}$ Evidently, all linear combinations of characteristic functions (i.e., the so-called simple or step-functions) of the form $\sum_{i \leq n} c_{i} \cdot \mathbf{1}_{B_{i}}$ with measurable sets $B_{1}, \cdots, B_{n}$ in $\mathcal{B}$ and scalars $c_{1}, \cdots, c_{n}$ in $\mathbb{R}$ are also members of the concrete von Neumann algebra. The other measurable functions in $\boldsymbol{Q}_{\infty}(\mathscr{X}, \mathcal{B}, \mathbf{P})$ are obtained as (essential norm-based) limits of converging sequences of simple functions.

In the same spirit, it is easy to see that any measurable function $f \in \boldsymbol{Q}_{\infty}(\mathscr{X})$ with finitely many values has a spectral decomposition, represented by the weighted sum $f=$ $\sum_{i \leq n} c_{i} \cdot \mathbf{1}_{\llbracket f=c_{i} \rrbracket}$ of projection quantities, induced by the measurable partition $\left\{\llbracket f=c_{1} \rrbracket, \llbracket f=c_{2} \rrbracket, \cdots, \llbracket f=c_{n} \rrbracket\right\}$ of $\mathscr{X} .^{28}$ The foregoing spectral representation of quantity $f$ readily extends to a countable spectral resolution, also having the form of the weighted-sum $f=\sum_{c \in \operatorname{Spec}(f)} c \cdot \mathbf{1}_{\llbracket f=c \rrbracket}$, where

\footnotetext{
${ }^{26}$ In general, the set of projections $\mathfrak{A}_{p}$ in a von Neumann algebra $\mathfrak{A}$ is a complete lattice under the partial ordering $P \leq Q$ iff $P \bullet Q=Q \bullet P=P$, and lattice operations $P \vee Q=P+Q-P \bullet Q$ and $P \wedge Q=P \bullet Q$ in the commutative case. The lattice $\mathfrak{A}_{p}$ of projections of the von Neumann algebra $\mathfrak{A}$ is Boolean if and only if the algebra is commutative. In particular, the algebra of projections in $\mathbf{\Omega}_{\infty}(\mathscr{X}, \mathcal{B}, \mathbf{P})$ is isomorphic to the quotient Boolean sigma algebra, obtained from $\mathcal{B}$ by quotienting with the equivalence $B \sim B^{\prime}$ iff $\mathbf{P}\left(\left(B-B^{\prime}\right) \cup\left(B^{\prime}-B\right)\right)=0$.

${ }^{27}$ In quantum measurement theory this is the most compelling reason for replacing propositions by projections.

${ }^{28}$ Here, as before, the expression $\llbracket f=c_{i} \rrbracket$ denotes the measurable set of all points $x$ satisfying $f(x)=c_{i}$ with $1 \leq i \leq n$.
}

$\sum_{c \in \operatorname{Spec}(f)} \mathbf{1}_{\llbracket f=c \rrbracket}=\mathbf{1}$. This leads at once to derived-quantity representations encoded by $g(f)=\sum_{c \in \operatorname{Spec}(f)} g(c) \cdot \mathbf{1}_{\llbracket f=c \rrbracket}$ for any real-valued Borel measurable function $g$ on $\operatorname{Spec}(f)$ and to joint representations of the form $g\left(f, f^{\prime}\right)=\sum_{c, c^{\prime}} g\left(c, c^{\prime}\right)$. $\mathbf{1}_{\llbracket f=c, f^{\prime}=c^{\prime} \rrbracket}$.

\subsection{Spectral representation of measurable quantities}

Based on the spectral mapping theorem, we can now clarify how projections can be used to represent any measurable quantity whatsoever. Let $Q$ be a quantity in a unital commutative von Neumann algebra $\mathfrak{A}$. Appealing to measurable function calculus, we may define a canonical projection quantity $\boldsymbol{\Pi}_{Q}(B)={ }_{d f} \mathbf{1}_{B}(Q)$ for any Borel measurable set $B$ in the Borel sigma algebra $\mathcal{B}_{\operatorname{Spec}(Q)}$ of measurable subsets of $\operatorname{Spec}(Q)$. Clearly, since the characteristic function $\mathbf{1}_{B}$ is a projection, the quantity $\boldsymbol{\Pi}_{Q}(B)$ (encoding algebraically the proposition " $Q$ takes its extant value in $B$ ") is also a projection, belonging to the von Neumann algebra $\mathfrak{A}(Q){ }^{29}$ It is assigned to the projection function $\mathbf{1}_{B}$ by the isomorphism $\mathbf{I}_{Q}$. We have now constructed a crucial projective representation map $\boldsymbol{\Pi}_{Q}: \mathcal{B}_{\operatorname{Spec}(Q)} \longrightarrow \mathfrak{A}(Q)_{p}$, called the projection-valued measure (acronymed PVM) associated with quantity $Q$ that allows us to look at quantities from a new point of view.

In general, a PVM is any mapping $\boldsymbol{\Pi}: \mathcal{B} \longrightarrow \mathfrak{A}_{p}$ on a measurable space $\langle\mathscr{X}, \mathcal{B}\rangle$ that assigns to each measurable set $B$ in $\mathcal{B}$ a unique projection (i.e., two-valued quantity) $\Pi(B)$ belonging to the algebra $\mathfrak{A}$ in such a way that the following measure-theoretic conditions hold for all measurable sets $B, B^{\prime}$ in $\mathcal{B}$ and for all sequences of pairwise disjoint measurable sets $B_{1}, B_{2}, \cdots$ in $\mathcal{B}$ :

(i) $\boldsymbol{\Pi}(\emptyset)=\mathbf{0}$ and $\boldsymbol{\Pi}(\mathscr{X})=1$.

(ii) $B \subseteq B^{\prime} \Longrightarrow \Pi(B) \leq \Pi\left(B^{\prime}\right)$.

(iii) $\Pi\left(B_{1}+B_{2}+\cdots\right)=\Pi\left(B_{1}\right)+\Pi\left(B_{2}\right)+\cdots$, where the countable sum on the right-hand side is interpreted as the limit of the sequence of partial sums in the so-called weak topology of $\mathfrak{A} .^{30}$

(iv) $\Pi\left(B \cap B^{\prime}\right)=\Pi(B) \cdot \Pi\left(B^{\prime}\right)$.

(v) $B \cap B^{\prime}=\emptyset \Longrightarrow \Pi(B) \cdot \Pi\left(B^{\prime}\right)=\mathbf{0}$.

Note that projection-valued measures behave like special probability measures, except that their values are not numbers but projection quantities in a von Neumann algebra. More significantly perhaps, PVMs are not only important and interesting in their own right, one can actually integrate functions with respect to them. Specifically, the so-called spectral representation theorem states that to each quantity $Q$ in a von

\footnotetext{
${ }^{29}$ Note that for $f \in \mathbf{Q}_{\infty}(\mathscr{X})$, we have $\boldsymbol{\Pi}_{f}(B)=\mathbf{1}_{\llbracket f \in B \rrbracket}$, where the expression $\llbracket f \in B \rrbracket$ denotes the measurable subset $\sum_{c \in B} \mathbf{1}_{\llbracket f=c \rrbracket}=f^{-1}(B)=\{x \mid f(x) \in B\}$ of $\operatorname{Spec}(f)$.

${ }^{30}$ Recall that in commutative settings, the weak topology is defined by the weak $\operatorname{limit}_{\text {wim }} \rightarrow \infty Q_{n}=Q$ if and only if $\lim _{n \rightarrow \infty} \mathcal{S}\left(Q_{n}-Q\right)=0$ for all states $\mathcal{S}$.
} 
Neumann algebra $\mathfrak{A}$ there corresponds a unique projectionvalued measure $\Pi_{Q}$ such that the following spectral integral representations hold

$$
Q=\int_{\operatorname{Spec}(Q)} x \cdot \boldsymbol{\Pi}_{Q}(\mathrm{~d} x) \text { and } f(Q)=\int_{\operatorname{Spec}(Q)} f(x) \cdot \boldsymbol{\Pi}_{Q}(\mathrm{~d} x)
$$

for any essentially bounded measurable function $f$ : $\operatorname{Spec}(Q) \longrightarrow \mathbb{R}$. In a function algebra setting, the foregoing spectral integral is defined in the usual way, starting from simple functions and then moving up to all measurable functions by a limit construction. In particular, the characteristic functions satisfy the following conditions:

(i) $\int_{\operatorname{Spec}(Q)} \mathbf{1}_{B}(x) \cdot \boldsymbol{\Pi}_{Q}(\mathrm{~d} x)=\boldsymbol{\Pi}_{Q}(B)$.

(ii) The constant function $k_{1}$ gives the resolution of the unit $\int_{\operatorname{Spec}(Q)} \boldsymbol{\Pi}_{Q}(\mathrm{~d} x)=\mathbf{1}$.

(iii) For the simple function $f=\sum_{1 \leq i \leq n} c_{i} \cdot \mathbf{1}_{B_{i}}$ with pairwise disjoint measurable subsets $B_{1}, B_{2}, \cdots B_{n}$ we have $\int_{\operatorname{Spec}(Q)} \mathbf{1}_{B}(f(x)) \cdot \boldsymbol{\Pi}_{Q}(\mathrm{~d} x)=\sum_{1 \leq i \leq n} c_{i} \cdot \boldsymbol{\Pi}_{Q}\left(B_{i}\right)$.

So what are these spectral representations of quantities good for? The single most important consequence of writing quantities in their canonical spectral form is in relating quantities to their associated measurement operations. For example, by appealing to the spectral representation result the physicist may view the position quantity $Q$ of a free Newtonian particle moving in a straight line in terms of its corresponding projection-valued measure $\boldsymbol{\Pi}_{Q}$ that outputs value $\boldsymbol{\Pi}_{Q}(B)=1$, when the particle is in region $B$ and value 0 when it is not, for any measurable spatial region $B$. Thus, to measure the particle's exact position means asking and answering all questions of the form "Is the particle in region $B$ ?" at the same time and then moving on to perform the corresponding binary yes-no measurements. In concordance with the spectral representation of the position quantity $Q$, exactly one answer will be "yes", when the particle is actually in region $B$. However, it is important to realize that the particle's position at any given moment depends on its actual physical state, and therefore one cannot tell tell ahead of time where the particle really is. Nevertheless, as we shall see shortly, measurement theory predicts that the particle will be in region $B$ with probability $\mathcal{S}\left(\Pi_{Q}(B)\right)$, given that at that time it will be in the physical state represented by $\mathcal{S}$. Now, since classical mechanics treats particles as deterministic systems, its state $\mathcal{S}$ is deterministic, specified by the particle's position and momentum values, and therefore the probability value will be 1 precisely when the position coodinate falls into region $B$.

In this manner, PVMs explain how sharp measurements are possible in terms of ideal binary measurements. On this scenario, any quantity $Q$ can be looked at from the point of view of its (infinitely long) disjunction $\llbracket Q=c \rrbracket$ or $\llbracket Q=c^{\prime} \rrbracket$ or $\cdots$ propositions, algebraically represented by a weighted sum (or integral) of projection quantities $\boldsymbol{\Pi}_{\{c\}}, \boldsymbol{\Pi}_{\left\{c^{\prime}\right\}}, \cdots$, assigned to singletons $\{c\},\left\{c^{\prime}\right\}, \cdots$.. The question now arises as to how can we make the PVM approach more realistic.

Because the exact values of quantities (and states) are generally inaccessible to direct observation, that is to say, the experimenter is denied knowledge of sharp propositions of the form $\llbracket f=c \rrbracket$, a crucial epistemic strategy is a passage from the underlying point $\operatorname{set} \operatorname{Spec}(Q)$ framework to its higherlevel Borel algebra $\mathcal{B}_{\operatorname{Spec}(Q)}$ setting. Owing to this conceptual move from equational propositions $\llbracket f=c \rrbracket$ to more general and less informative set-theoretic propositions of the form $\llbracket f \in B \rrbracket$, the experimenter will certainly know whether or not $\llbracket f \in B \rrbracket$ holds for appropriately large Borel sets $B$. Unfortunately, stochastic systems possess behaviors in which the experimenter does not strictly know the propositions of the form $\llbracket f \in B \rrbracket$ either, but (s)he may know that the outcome $c$ is more likely to be in some parts of $\operatorname{Spec} Q$ than others. As we will see later, in this context the experimenter's epistemic access to measurement outcomes is encoded by probability values of the form $\mathbf{P}(\llbracket f \in B \rrbracket)$, thought of as the probability that the extant value of $f$ is in the Borel set $B$.

As a matter of fact, in quantum theory of measurement it has become customary to go one step further in the direction of fuzzifying or smearing the measurement results by replacing all measurands $Q$ with spectral measures of a highly general form $\boldsymbol{\Pi}: \mathcal{B} \longrightarrow \mathfrak{A}$ on an arbitrary probability space $\langle\mathscr{X}, \mathcal{B}, \mathbf{P}\rangle$ with values in the target system's quantity algebra $\mathfrak{A}$. These so-called (normalized) positive operator-valued measures (with a generally accepted acronym POVM) satisfy the following conditions for all measurable sets $B \in \mathcal{B}$ and for all countable sequences of pairwise disjoint sets $B_{1}, B_{2}, \cdots$ in $\mathcal{B}$ :

(i) $\boldsymbol{\Pi}(\emptyset)=\mathbf{0}$ and $\boldsymbol{\Pi}(\mathscr{X})=1$.

(ii) $\boldsymbol{\Pi}(B) \geq \mathbf{0}$.

(iii) $\boldsymbol{\Pi}\left(B_{1}+B_{2}+\cdots\right)=\boldsymbol{\Pi}\left(B_{1}\right)+\boldsymbol{\Pi}\left(B_{2}\right)+\cdots$, where the sum on the right-hand side is again interpreted as the limit of the sequence of partial sums in the weak topology of $\mathfrak{A}$.

In quantum physics, positive operator-valued measures are regularly used in modeling the (tail-end) statistical aspects of outcomes of quantum measurement procedures, independently of the physical structure of measuring instruments. The probability that the measurement outcome belongs to a Borel set is given by the expectation value of the POVM's quantity. In the class of discrete projective (binary) measurements, each quantity $Q$ has a spectral decomposition $Q=\sum_{c \in \operatorname{Spec}(Q)} c \cdot \boldsymbol{\Pi}_{c}$, so that the probability of obtaining outcome $c$ is given by the expectation $\mathcal{S}\left(\boldsymbol{\Pi}_{c}\right)$ with respect to the system's extant state $\mathcal{S}$ in $\mathbf{S}(\mathfrak{A})$. We hasten to add that the foregoing POVM-based statistical model of measurement says nothing about the target system's post-measurement state, i.e., the system's posterior state, conditioned by the observed measurement outcome. Not surprisingly, the statistical model provides the least detailed characterization of measurement in that it accounts only for the target system's measurement outcomes that can 
be manipulated and fed into various postmeasurement processors.

Next, we shall need to understand how abstractly given states of a system under consideration may be interpreted probabilistically. The state space framework provides a fundamental theorem that addresses this issue. In detail, each quantity $Q$ in a von Neumann algebra $\mathfrak{A}$ determines an affine mapping of the form

$$
\mathbf{S}(\mathfrak{I}) \stackrel{\Pi_{Q}^{*}}{\longrightarrow} \mathbf{I P}(\operatorname{Spec}(Q)),
$$

defined by $\boldsymbol{\Pi}_{Q}^{*}(\mathcal{S})=_{d f} \mathbf{P}_{Q, \mathcal{S}}$ for all states $\mathcal{S}$ in $\mathbf{S}(\mathfrak{A})$, where the unique probability measure $\mathbf{P}_{Q, \mathcal{S}}$ satisfies the classical expectation formula

$$
\mathcal{S}(f(Q))=\int_{\operatorname{Spec}(Q)} f(x) \mathbf{P}_{Q, \mathcal{S}}(\mathrm{d} x)
$$

for all measurable functions $f: \operatorname{Spec}(Q) \longrightarrow \mathbb{R}$. The equality $\mathbf{P}_{Q, \mathcal{S}}=\mathcal{S} \circ \boldsymbol{\Pi}_{Q}$ (in which $\boldsymbol{\Pi}_{Q}$ is the projection-valued measure associated with $Q$ ) gives a direct definition of the abovespecified probability measure. In particular, for any a Borel subset $B$ of $\operatorname{Spec}(Q)$ we have the probability

$$
\mathbf{P}_{Q, \mathcal{S}}(B)=\mathcal{S}\left(\boldsymbol{\Pi}_{Q}(B)\right)=\mathcal{S}\left(\mathbf{1}_{B}(Q)\right)
$$

stating that $Q$ 's extant value is in the subset $B$, given that the system is in a physical state encoded by $\mathcal{S}$. Actually, the above-mentioned fundamental theorem says more: To each state-channel $\mathbf{M}^{*}$ there corresponds a unique transition probability (Markov kernel) $\mathbf{T}_{\mathbf{M}}$ giving a commutative diagram

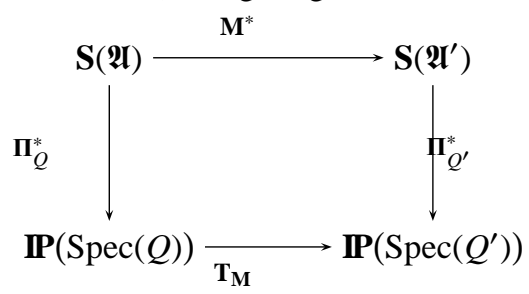

with $\boldsymbol{\Pi}_{Q^{\prime}}^{*} \circ \mathbf{M}^{*}(\mathcal{S})=\mathbf{T}_{\mathbf{M}} \circ \boldsymbol{\Pi}_{Q}^{*}(\mathcal{S})$ for all states $\mathcal{S}$ in $\mathbf{S}(\mathfrak{A})$ and hence $\mathbf{P}_{Q^{\prime}, \mathbf{M}^{*}(\mathcal{S})}(B)=\left[\mathbf{T}_{\mathbf{M}}\left(\mathbf{P}_{Q, \mathcal{S}}\right)\right](B)$ for all Borel subsets $B$ of $\operatorname{Spec}\left(Q^{\prime}\right)$.

The reader will surely notice that in parallel with the earlier discussed passage from abstract measurable quantities to classical random variables we have just introduced the dual idea of reasoning about abstract states in terms of concrete probability measures. Thus, the earlier posed question is taken up again: Why bother with abstract states, when probabilitiy measures seem to suffice? Because states occupy a unifying position in the representation of all kinds of physical states, including those of quantum systems, where the fundamental theorem about mapping states to classical probability measures fails. In particular, quantum measurement relies on noncommutative probability theory, which is formulated exclusively in the language of algebraic states. For a systematic treatment of transition probabilities in a rather general setting see [6].
The abstract framework of states includes the following general versions of basic statistical indicators for all state states $\mathcal{S}$ :

(i) Expectation of $Q$ : $\mathcal{S}(Q)=d_{d f} \int_{\operatorname{Spec}(Q)} x \mathbf{P}_{Q, \mathcal{S}}(\mathrm{d} x)$.

(ii) Variance of $Q: \operatorname{Var}_{\mathcal{S}}(Q)={ }_{d f} \mathcal{S}\left(Q^{2}\right)-\mathcal{S}(Q)^{2}$. By definition, the so-called ideal measurement operation $\mathbf{M}$ satisfies the conservation law of variance: $\operatorname{Var}_{\mathcal{S}}(Q)=$ $\operatorname{Var}_{\mathbf{M}^{*}(\mathcal{S})}\left(Q^{\uparrow}\right)$. Variance is particularly useful in assessing the fidelity of state-channels.

It is time we had some concrete examples of measurement models.

\section{Algebraic MOdELS OF ElEMENTARY MEASUREMENT} PROCEDURES

In this section we survey a number of concrete examples of classical measurement, alluded to in the previous sections. We emphasize at the outset that we do not have space to present a complete analysis of all examples. Each example merits considerable study in its own right and we intend to pursue such studies in the future. Here our goal is to show how algebraic constructions capture the decisive structures of measurement operations. The examples of elementary measurement we shall cover in this section fall naturally into three relatively disjoint classes: direct, indirect and joint measurements.

The simplest and earliest types of direct measurement (e.g., length and mass measurements) are based on the principle of direct comparison. A direct-comparison process relies on comparing the measurand's values with those of its associated pointer quantity of the same dimension and in a comparable range. It is convenient to begin with the basic idea of a static measurement of length.

\subsection{Classical comparison-based length measurement}

In this example the measuring device is the familiar meter stick or ruler with a calibrated equally-spaced mark for, say, each millimeter along the ruler's total length of one meter (i.e., $10^{3}$ millimeters). We know that the (approximate) length of, for example, a flagpole is found by placing the meter ruler alongside it, starting from one end and then repeatedly stepping it off, until the flagpole's other end is reached. At that point the scale mark that is closest to the flagpole's other end is read and added to the millimeters obtained from the total number of collinear ruler steppings along the flagpole's entire length. An earlier version of the length measurement structures presented here is discussed in [3]. ${ }^{31}$

\footnotetext{
${ }^{31}$ Today's technology is blessed with many types of non-contact length measurements, including ultrasound, laser and radar-based instruments. These technologies have taken over the task of length measurement from simple-minded meter stick devices. In the algebraic approach to follow we will discuss only the simplest cases of measurement, where a suitable instrument scale must be read to obtain the measurement result.
} 
Suppose we wish to measure the length $\ell$ of a flagpole with a meter ruler. So $\ell$ is a measurable quantity (representable by a random variable), where we take the closed real interval $\operatorname{Spec}(\ell)=[0, L]$ to be its value set with a sufficiently large $L$. Now the basic modeling idea is to regard the flagpole as our target system and associate with it the von Neumann algebra $\boldsymbol{\mathfrak { L }}_{\infty}([0, L])$ of quantities, isomorphic to the abstract von Neumann algebra $\mathfrak{A}(\ell)$, generated by $\ell$ and $1 .^{32}$ Since this algebra contains all quantities that are derivable from the length quantity $\ell$, it follows that their values can automatically be calculated from the extant value of $\ell$.

Having assigned a quantity algebra to the flagpole, we now construct an algebra for the ruler. We know that in order to obtain information about the flagpole's actual length, the experimenter will have to get it from stepping off the ruler against the flagpole and from reading the numerals at the ruler's discrete markings. Clearly, the fidelity of this measurement operation depends on the ruler's resolution and accuracy. ${ }^{33}$ One of the most intuitive ways to proceed is to introduce a discrete-valued pointer quantity $\ell^{\uparrow}$ that characterizes the ruler, so that $\ell^{\uparrow}$ 's spectrum $\operatorname{Spec}\left(\ell^{\uparrow}\right)$ is specified by the ruler's reading scale, i.e., by the set of points in $[0, L]$ corresponding to the millimeter marks, encoded by parameter $\varepsilon>0$ :

$$
\operatorname{Spec}\left(\ell^{\uparrow}\right)=[0, L]_{\varepsilon}=d f \varepsilon \mathbb{N} \cap[0, L],
$$

where the discrete lattice $\varepsilon \mathbb{N}=\{0, \varepsilon, 2 \varepsilon, 3 \varepsilon, \cdots\}$ denotes the uniform grid of equally spaced points one millimeter apart (i.e., the step-size $\varepsilon$ represents the distance of one millimeter), capturing the ruler's discrete length structure. We are now able to introduce the ruler's finite-dimensional von Neumann algebra $\mathfrak{L}_{\infty}\left([0, L]_{\varepsilon}\right)$, isomorphic to the von Neumann algebra $\mathfrak{A}\left(\ell^{\uparrow}\right)$, in which the pointer quantity corresponds to the canonical identity function $\mathfrak{J}$, defined by $\mathfrak{J}(x)=x$ for all $x$ in $[0, L]_{\varepsilon}$.

The measurand's values are related to those of its pointer quantity by the round-off map $\mathfrak{R}_{\varepsilon}:[0, L] \longrightarrow[0, L]_{\varepsilon}$, defined by $\mathfrak{R}_{\varepsilon}(c)={ }_{d f} k \varepsilon$, if $k \varepsilon-\frac{1}{2} \leq c<k \varepsilon+\frac{1}{2}$ for all $k \geq 1$, and $\mathfrak{R}_{\varepsilon}(c)=0$ for all $c<\frac{1}{2} \varepsilon$. Intuitively, the round-off map $\mathfrak{R}_{\varepsilon}$ assigns to each possible value $c$ of measurand $\ell$ the unique point $\mathfrak{R}_{\varepsilon}(c)$ in $[0, L]_{\varepsilon}$ that is closest to it. ${ }^{34}$. We also have the obvious inclusion map $\mathfrak{J}_{\varepsilon}:[0, L]_{\varepsilon} \longrightarrow[0, L]$ that sends the points of the discrete subset $[0, L]_{\varepsilon}$ to the continuum value set $[0, L]$. We know from functional analysis that the abovedefined round-off and inclusion maps induce two quantitychannels

\footnotetext{
${ }^{32}$ As discussed in Section 4 , the interval $[0, L]$ is conveniently viewed as a probability space, where the Boolean algebra $\mathcal{B}$ is generated by the open subsets of $[0, L]$ and in simple cases the probability measure $\mathbf{P}$ is just the standard Lebesgue measure thereon.

${ }^{33}$ Even in the most general context of measurement, reading the measurement results relies on being able to discriminate between a finite or a countable set of possible pointer quantity values, defined by a coarse-grained variant of the measurand's spectrum. Due to the discrete nature of most measurements, the hallmark of the algebraic approach is to assign discrete models to measuring instruments and try to represent the target system by a continuum structure. In many applications, continuum models can be viewed as category-theoretic inverse limits of directed sequences of discrete models.

${ }^{34} \mathrm{~A}$ concrete analysis of rounded data may be found in [1].
}

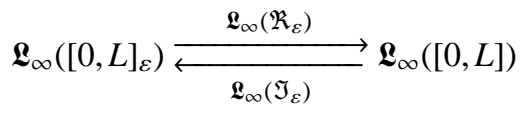

defined by the injective algebra homomorphism $\boldsymbol{Q}_{\infty}\left(\mathfrak{R}_{\varepsilon}\right)(h)={ }_{d f} h \circ \mathfrak{R}_{\varepsilon}$ for all functions $h$ on $\left.[0, L]_{\varepsilon}\right)$ and by the surjective homomorphism $\mathfrak{L}_{\infty}\left(\mathfrak{J}_{\varepsilon}\right)(g)=_{d f} g \circ \mathfrak{J}_{\varepsilon}$ for all functions $g$ on $[0, L]$. Since the first channel represents length measurement, we introduce the simplified notation $\mathbf{M}_{\varepsilon}$ for $\mathfrak{L}_{\infty}\left(\mathfrak{R}_{\varepsilon}\right)$. The second channel is just a left-inverse projection map of norm one, also known as a statistically significant transition expectation $\mathbf{E}\left(\cdot \mid \mathfrak{R}_{\varepsilon}\right)$.

Note that in the foregoing definitions of quantity algebras we did not include any details of exactly how the ruler interacts with the flagpole. Instead, we considered only the way propositions of the form $\llbracket \ell^{\uparrow}=k \rrbracket$ about the pointer quantity values might be translated by the quantity-channel into propositions of the form $\llbracket \ell=c \rrbracket=\{x \mid \ell(x)=c\}$ about the measurand's values.

As an intermediate translation step, we rephrase these propositions in terms of projection quantities $\mathbf{1}_{\llbracket \ell^{\uparrow}=k \varepsilon \rrbracket}$ and $\mathbf{1}_{\llbracket \ell=c \rrbracket}$ respectively. It is easy to see that the pointer quantity has the spectral representation $\ell^{\uparrow}=\varepsilon \cdot \mathbf{1}_{\llbracket \ell^{\uparrow}=\varepsilon \rrbracket}+2 \varepsilon \cdot \mathbf{1}_{\llbracket \ell^{\uparrow}=2 \varepsilon \rrbracket}+$ $\cdots$ and similarly, the measurand's estimate is given by the resolution $\widehat{\ell}(c)=\sum_{k \geq 1} \mathfrak{R}_{\varepsilon}(c) \cdot \mathbf{1}_{\llbracket k \varepsilon-\frac{1}{2} \leq c<k \varepsilon+\frac{1}{2} \rrbracket}$ for all $c \geq \frac{1}{2} \varepsilon$, and 0 otherwise.

Now we can immediately conclude from the foregoing analysis of length measurement that the quantity-channel $\mathbf{M}_{\varepsilon}$ sends the ruler's projections (representing pointer propositions) to the flagpole's projections (encoding the measurand's approximation) as follows:

$$
\mathbf{M}_{\varepsilon}\left(\mathbf{1}_{\llbracket \ell \uparrow=k \varepsilon \rrbracket}\right)=\mathbf{M}_{\varepsilon}\left(\mathbf{1}_{\{k \varepsilon\}}\right)=\mathbf{1}_{\left[k \varepsilon-\frac{1}{2}, k \varepsilon+\frac{1}{2}\right)}
$$

for all $k$. It is clear that a ruler-based length measurement is far from being ideal. Indeed, based on the assignment $\mathbf{M}_{\varepsilon}\left(\ell^{\uparrow}\right)=\mathbf{M}_{\varepsilon}(\mathfrak{J})=\widehat{\ell}$, ruler-reading provides only a stepfunction approximation $\widehat{\ell}$ of $\ell$, constant on half-open intervals $\left[k \varepsilon-\frac{1}{2}, k \varepsilon+\frac{1}{2}\right)$. In general we have $\mathbf{M}_{\varepsilon}(h)=\sum_{k \geq 1} h(k \varepsilon)$. $\mathbf{1}_{\left[k \varepsilon-\frac{1}{2}, k \varepsilon+\frac{1}{2}\right)}$ for all $h:[0, L]_{\varepsilon} \longrightarrow \mathbb{R}$.

Of course, if an alternate meter ruler is marked at every half millimeter along its length, then its resolution becomes significantly better and therefore the measurand's step-function approximation $\mathbf{M}_{\frac{\varepsilon}{2}}\left(\ell^{\uparrow}\right)$ will also be correspondingly more finegrained. In the limit, when the distance $\varepsilon$ goes to zero, a maximally "good" resolution characterizes an ideal measurement with $\mathbf{M}_{0}\left(f\left(\ell^{\uparrow}\right)\right)=f(\ell)$ for all functions $f$ defined on $\operatorname{Spec}(\ell)$. Measurement results based on these two meter rulers are shown in Figure 6. The diagonal line in the schematic diagram indicates the ideal measurement of length and the two step-functions correspond to the measurand's approximations obtained by a ruler having a mark for each millimeter and another ruler having a mark for each half millimeter. 


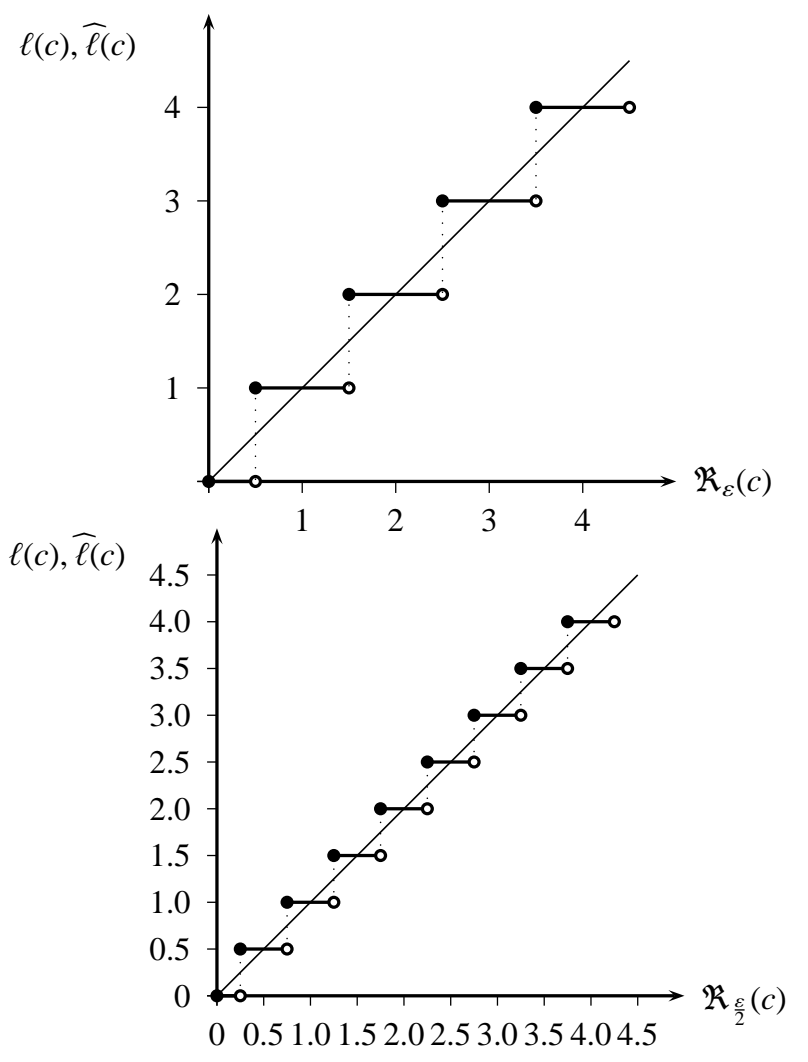

Fig. 6. Ruler-based step-function approximations of length quantity $\ell$.

Length measurement can also be reasoned about in terms of state-channels, having the form

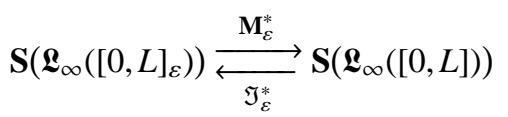

where $\mathfrak{J}_{\varepsilon}^{*}$ is an extension map that, roughly speaking, extends discrete probability distributions on $[0, L]_{\varepsilon}$ to optimally approximating continuous distributions on $[0, L]$. This process is actually a special case of probability kinematics.

Recall from the end of Section 4 that all states in the state space $\mathbf{S}\left(\boldsymbol{\mathfrak { Q }}_{\infty}([0, L])\right)$ are representable by probability measures belonging to $\mathbf{I P}([0, L])$. Importantly, the state-channel $\mathbf{M}_{\varepsilon}^{*}$ is represented by a unique transition probability of the form

$$
\mathbf{I P}([0, L]) \stackrel{\mathbf{T}_{\varepsilon}^{*}}{\longrightarrow} \mathbf{I P}\left([0, L]_{\varepsilon}\right)
$$

that sends all probability distributions on $[0,1]$ to some probability distributions on $[0, L]_{\varepsilon}$. It is well known that the abovedisplayed transition probability is equivalent to the channel distribution $\mathbf{T}_{\varepsilon}^{\prime *}:[0, L] \longrightarrow \mathbf{I P}\left([0, L]_{\varepsilon}\right)$, where the number $\left\{\left[\mathbf{T}_{\varepsilon}^{\prime *}\right](c)\right\}(\{k \varepsilon\})$ denotes the probability that the flagpole's length measured by the ruler is $k \varepsilon$ millimeters, given that its actual length is $c$.

In deterministic situations it is natural to assume that the flagpole's extant state $\mathcal{S}$ is pure, meaning the flagpole's actual length is a definite (albeit unknown) real number, say $c$, in that state. Thus, $\mathcal{S}$ may be represented by a Dirac probability measure $\boldsymbol{\delta}_{c}$. In this case we have $\mathcal{S}\left(\mathbf{1}_{\llbracket \ell=c \rrbracket}\right)=\mathbf{P}_{\ell, \mathcal{S}}(\llbracket \ell=$ $c \rrbracket)=1$ precisely when the flagpole's length is actually $c$, and $\mathcal{S}\left(\mathbf{1}_{\llbracket \ell=c \rrbracket}\right)=0$ otherwise. Intuitively, a pure state ensures a probabilistic certainty about the extant value of $\ell$. Now, since the state-channel $\mathbf{M}_{\varepsilon}$ is also pure (in view of being determined by the discretization map $\mathfrak{R}_{\varepsilon}$ ), it transfers the flagpole's deterministic state to the ruler's deterministic state, so that the ruler's readout is exactly $\mathfrak{R}_{\varepsilon}(c)$ in that state, captured by $\boldsymbol{\delta}_{\mathfrak{R}_{\varepsilon}(c)}$. Thus, by the fundamental state representation theorem we have

$$
\begin{gathered}
\mathcal{S}\left(\mathbf{1}_{\llbracket \ell^{\uparrow}=\mathfrak{R}_{\varepsilon}(c) \rrbracket}\right)=\mathbf{P}_{\ell^{\uparrow}, \mathbf{M}_{\varepsilon}^{*}(\mathcal{S})}\left(\llbracket \ell^{\uparrow}=\mathfrak{R}_{\varepsilon}(c) \rrbracket\right) \\
=\left[\mathbf{T}_{\varepsilon}^{*}\left(\mathbf{P}_{\ell, \mathcal{S}}\right)\right]\left(\llbracket \ell^{\uparrow}=\mathfrak{R}_{\varepsilon}(c) \rrbracket\right)=1,
\end{gathered}
$$

if $\mathbf{P}_{\ell, \mathcal{S}}(\llbracket \ell=c \rrbracket)=1$, and 0 otherwise.

We mention in passing the case of repeated length measurement. For example, if the flagpole's length is measured twice, then the representing state-channel has the form

$$
\mathbf{S}\left(\boldsymbol{L}_{\infty}([0, L])\right) \stackrel{\mathbf{M}_{\varepsilon}^{*(2)}}{\longrightarrow} \mathbf{S}\left(\mathfrak{L}_{\infty}\left([0, L]_{\varepsilon} \times[0, L]_{\varepsilon}\right)\right)
$$

where in deterministic situations the channel $\mathbf{M}_{\mathcal{\varepsilon}}^{*(2)}$ sends each Dirac state $\boldsymbol{\delta}_{c}$ (representing the flagpole's actual length) to the average distribution $\frac{1}{2}\left[\boldsymbol{\delta}_{\mathfrak{R}_{\varepsilon}(c)}+\boldsymbol{\delta}_{\mathfrak{R}_{\varepsilon}^{\prime}(c)}\right]$ obtained from two independently performed measurements for all values $c$. Clearly, in this measurement there are two probabilistically independent pointer quantities $\ell_{1}^{\uparrow}$ and $\ell_{2}^{\uparrow}$ together with two independent around-off maps. The situation of $n$-fold repeated measurement processes is treated similarly, where the probability distribution of measurement outcomes has the form $P=\sum_{k \geq 1} \boldsymbol{\delta}_{k \varepsilon}$.

As for nondeterministic cases, when the flagpole's actual length is uncertain (due to various perturbation factors), we can safely assume that there is a normal probability distribution that correctly describes the flagpole's geometric condition. As expected, flagpole states characterized by normal distributions are transformed by the state-channel $\mathbf{M}^{*}$ into ruler states, having the form of binomial distributions that discretely approximate the continuum input distributions. A state-channel transfer from a continuum distribution to an optimal discrete distribution is illustrated in Figure 7.

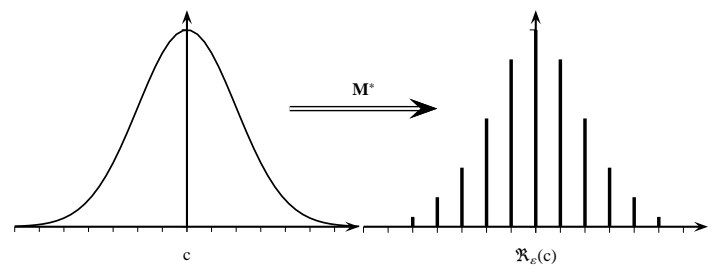

Fig. 7. State-channel transfer of normal ditributions to approximating binomial distributions.

States represented by normal distributions usually arise from measurements that are repeated arbitrarily many times under 
the same or exchangeable conditions. In this case the measurement process involves an infinite sequence $\ell_{1}^{\uparrow}, \ell_{2}^{\uparrow}, \cdots$ of probabilistically independent random variables, whose Cartesian product value space $[0, L]_{\varepsilon}^{\mathbb{N}}=d_{d f}[0, L]_{\varepsilon} \times[0, L]_{\varepsilon} \times \cdots$ with its sigma Boolean algebra of cylinder sets induces a quantitychannel of the form

$$
\mathbf{S}\left(\boldsymbol{\mathfrak { Q }}_{\infty}([0, L])\right) \stackrel{\mathbf{M}_{\varepsilon}^{* N}}{\longrightarrow} \mathbf{S}\left(\mathfrak{Q}_{\infty}\left([0, L]_{\varepsilon}\right) \otimes \mathfrak{Q}_{\infty}\left([0, L]_{\varepsilon}\right) \otimes \cdots\right)
$$

sending, roughly speaking, the flagpole's probability distribution (representing its extant state) to a joint probability distribution on $[0, L]_{\varepsilon}^{\mathbb{N}}$.

For the balance of this subsection we wish to emphasize that the algebraic formalism presented above is also applicable to direct measurements of mass on an equal-arm balance scale. The target system is any small-size object with an unknown mass that is placed on the balance scale's right pan and a suitable set of standard weights with known masses is added to the left pan and adjusted until a perfect balance is achieved. For concreteness, we can assume that a balance scale can measure objects with masses up to 1000 grams and the smallest detectable mass for which there is a standard weight is 0.01 grams. As in the case of length measurement, instruments of this type provide only an optimal approximation of the measured object's actual mass. The theoretical limit for the resolution of a balance is set by the fact that, due to perturbations, the measuring instrument cannot be brought to absolute rest. In practice, the instrument's resolution is determined by the smallest mass of available standard weights.

\subsection{Indirect temperature measurement}

Microscopically, temperature is understood to be the average kinetic or thermal energy of a target thermodynamical system's atoms or molecules. Unfortunately, the temperature of thermodynamical systems cannot be measured directly. However, since higher degrees of temperature correspond to motions with greater amplitudes, thermometric property-based measuring instruments can record a certain manifestation of the increased energy of atoms or molecules. The most used macroscopic thermometric properties in thermometry are the volumetric expansion and electrical resistance of certain materials. Naturally, thermometers employ physical principles that link temperature changes to certain easy-to-measure thermometric property changes. In this way, thermometric quantities can be profitably utilized in measuring temperature indirectly. It is well known that indirect measurements have largely taken over the task of measurement from the basic and less effective direct measurement methods.

Perhaps the most popular physical principle used in thermometry is the expansion of liquids with increasing temperature. All of us are familiar with mercury-in-glass thermometers. Liquid mercury is encased in the glass bulb of a narrow glass capillary that expands to a greater volume when it gets hotter. The length of the mercury's expansion is measured by using a calibrated scale, etched in the stem of the thermometer. Importantly, the relationship between the temperature and the mercury column's height is linear over a limited temperature range, having the empirical law form

$$
\ell=\ell_{0}(1+\alpha \mathrm{T}),
$$

where $\ell$ denotes the length of the liquid mercury column in the thermometer's capillary at temperature $T, \ell_{0}$ is the nominal height of the column at zero temperature of Celsius, and $\alpha$ is the mercury's coefficient of linear expansion. Note that mercury-in-glass thermometers are passive instruments in that there is no external energy source needed for obtaining the measurement outputs.

Fundamental to mercury-in-glass thermometers and related measuring instruments is the thermometric map $£$ : $\operatorname{Spec}(T) \rightarrow \operatorname{Spec}(\ell)$, defined by $£(\tau)=\ell_{0}(1+\alpha \cdot \tau)$ for all $\tau$ in $\operatorname{Spec}(\mathrm{T})$, that assigns in a law-like manner to each temperature degree of the ambient thermodynamical system a unique mercury column length, realized by the measuring instrument after the two systems reach a thermal equilibrium. ${ }^{35}$ Along usual lines, the thermometric map $£$ induces the von Neumann algebra homomorphism

$$
\mathfrak{L}_{\infty}(\operatorname{Spec}(\ell)) \stackrel{\mathfrak{Q}_{\infty}(\mathfrak{f})}{\longrightarrow} \mathfrak{\mathfrak { L }}_{\infty}(\operatorname{Spec}(\mathrm{T}))
$$

that models temperature measurement, based on reading the mercury column's length values. In particular, the homomorphism $\mathfrak{L}_{\infty}(\mathfrak{E})$ sends projection $\mathbf{1}_{\llbracket \ell=c \rrbracket}$ (representing proposition $\llbracket \ell=c \rrbracket)$ to the projection $\mathbf{1}_{\llbracket \mathrm{T}=\frac{c-\ell_{0}}{\alpha \ell_{0}} \rrbracket}$ (representing proposition $\left.\llbracket \mathrm{T}=\frac{c-\ell_{0}}{\alpha \ell_{0}} \rrbracket\right)$. In ideal temperature measurements, the temperature quantity is fully determined by the length quantity, i.e., we have $\mathrm{T}=\mathfrak{L}_{\infty}(\mathfrak{f})\left(\frac{1}{\alpha \ell_{0}}\left(\ell-\ell_{0} \mathbf{1}\right)\right)$.

Since most real-world measurements are nonideal, the foregoing continuum model of measurement remains incomplete without its discretized counterpart. A complete model of indirect temperature measurement is given by the commutative diagram as shown below that includes the continuum part and the associated transformation of discrete readings of column length values to the correlated discrete values of temperature:

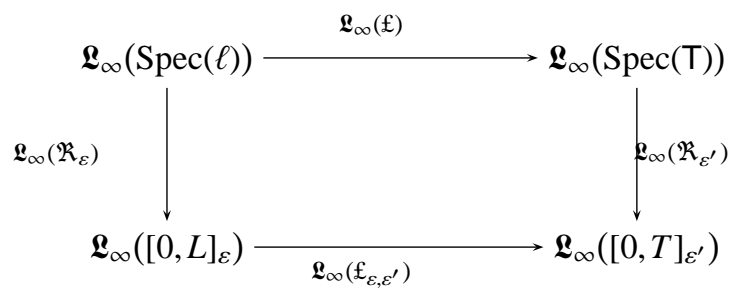

It is easy to see that the discrete homomorphism $\mathfrak{L}_{\infty}\left(\mathfrak{f}_{\varepsilon, \varepsilon^{\prime}}\right)$ sends the earlier discussed half-open intervals $\left[k \varepsilon-\frac{1}{2}, k \varepsilon+\frac{1}{2}\right)$

\footnotetext{
${ }^{35}$ The dynamics of mercury-in-glass thermometers in terms of heat transfers from system to thermometer is treated in [4].
} 
of column length to half-open intervals $\left[k \varepsilon^{\prime}-\frac{1}{2}, k \varepsilon^{\prime}+\frac{1}{2}\right)$ of temperature. Crucially, because thermometer readings provide only an estimate of the mercury column's actual length, the length-based derived measurement of temperature is also just an estimate of the 'true' values of T. Formally, we have $\widehat{\mathrm{T}}=\boldsymbol{\mathfrak { L }}_{\infty}(£)\left(\frac{1}{\alpha \ell_{0}}\left(\widehat{\ell}-\ell_{0} \mathbf{1}\right)\right)$.

A second common derived temperature measurement is performed by resistance temperature devices. Their design is based on the familiar principle that the electrical resistance of certain metals (e.g., platinum and nickel) increases with their increasing temperature. For a smaller range of temperature, the usual polynomial model relating resistance to temperature is safely approximated by the linear empirical law

$$
\mathrm{R}=\mathrm{R}_{0}(1+\alpha \mathrm{T})
$$

in which $R$ is the conductor's resistance at temperature $T$, $\mathrm{R}_{0}$ denotes the metal's nominal resistance at zero temperature (when it is immersed in melting ice), and $\alpha$ denotes the metal conductor's resistance-temperature coefficient. Importantly, resistance temperature devices are active measuring instruments, since the energy in the output signal comes from an external power souce, needed for measuring resistance. Typically, resistance thermometers consist of a coil of platinum wire wound on a ceramic insulator mounted into a steel tube, and connected to one arm of a Wheatstone bridge furnished with a small power source and a calibrated meter. Resistance temperature measurement is doubly indirect in that temperature changes are linearly related to resistance changes that are measured by a calibrated ohmmeter.

Today there is a large variety of thermometers that are based on considerably more sophisticated physical principles than the ones recalled above, such as bimetal thermometers, infra-thermometers, and pyrometers.

\subsection{Joint measurement of quantities}

We now move on to consider simultaneous measurements of several quantities. In a joint measurement of two quantities, information about their values is received from the respective pointer-quantities of two measuring instruments. A simple example is a simultaneous measurement of the position and angular velocity of a pendulum's bob. Here we are interested in the algebraic representation of measurements of this type.

Any pair $Q$ and $Q^{\prime}$ of measurands of a target system generating the von Neumann algebra $\mathfrak{A}\left(Q, Q^{\prime}\right) \subseteq \mathfrak{A}-$ can be viewed, thanks to the spectral mapping theorem, as random variables taking their values in their respective value sets $\operatorname{Spec}(Q)$ and $\operatorname{Spec}\left(Q^{\prime}\right)$. As one of the basic conclusions of the algebraic paradigm we note that the joint measurement of two measurands is meaningfully represented by the state-channel

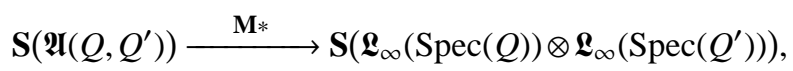

$$
\left[\mathbf{M}^{*}(\mathcal{S})\right](f \otimes g)=\int_{\operatorname{Spec} Q} \int_{\operatorname{Spec} Q^{\prime}} f(x) \cdot g(y) \mathbf{P}_{Q, Q^{\prime}, \mathcal{S}}(\mathrm{d} x, \mathrm{~d} y)
$$

for all real-valued functions $f$ on $\operatorname{Spec}(Q)$ and $g$ on $\operatorname{Spec}\left(Q^{\prime}\right)$, where $\mathbf{P}_{Q, Q^{\prime}, \mathcal{S}}$ denotes the probability measure on the product value space $\operatorname{Spec}(Q) \times \operatorname{Spec}\left(Q^{\prime}\right)$ representing state $\mathcal{S}$. Intuitively, the state-channel $\mathbf{M}^{*}$ of a joint measurement takes the system's state $\mathcal{S}$ as input and gives the probabily distribution $\mathbf{P}_{Q, Q^{\prime}, \mathcal{S}}$ on the measurand's values as output. As before, the identity maps $\mathfrak{J}$ and $\mathfrak{J}^{\prime}$ on value sets are the respective pointer quantities for $Q$ and $Q^{\prime}$.

There is an alternate equivalent representation of joint measurement that uses the dual quantity-channel $\mathbf{M}$. It renders the following diagram of marginal channels commutative:

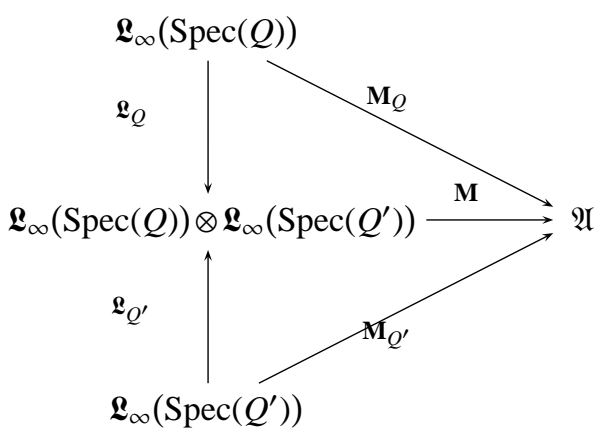

As expected, the marginal quantity-channels $\mathbf{M}_{Q}$ and $\mathbf{M}_{Q^{\prime}}$ included in the diagram represent the measurements of $Q$ and $Q^{\prime}$. By exactly the same reasoning as used in the previous section we obtain $\mathbf{M}(f \otimes \mathbf{1})=f(Q), \mathbf{M}(\mathbf{1} \otimes g)=g\left(Q^{\prime}\right)$, and hence $\mathbf{M}(f \otimes g)=(f \otimes g)\left(Q \otimes Q^{\prime}\right)$ for all real-valued functions $f, g$ defined on the value sets of $Q$ and $Q^{\prime}$. In particular, we have $\mathbf{M}(\mathfrak{J} \otimes \mathbf{1})=Q$ and $\mathbf{M}(\mathbf{1} \otimes \mathfrak{J})=Q^{\prime}$, suggesting that the joint measurement of $Q$ and $Q^{\prime}$ is treated as a case of ideal measurement. We already know that channels for nonideal joint measurements are based on discretizations of value sets $\operatorname{Spec}(Q)$ and $\operatorname{Spec}\left(Q^{\prime}\right)$, determined by the resolution and sensitivity of the measuring instruments selected for $Q$ and $Q^{\prime}$, respectively.

In joint measurements often it is of interest to determine how quantitiers $Q$ and $Q^{\prime}$ covary jointly. Recall that the covariance of $Q$ and $Q^{\prime}$ at state $\mathcal{S}$ is defined by $\operatorname{Covar}_{\mathcal{S}}\left(Q, Q^{\prime}\right)={ }_{d} f \mathcal{S}\left(Q \bullet Q^{\prime}\right)-\mathcal{S}(Q) \cdot \mathcal{S}\left(Q^{\prime}\right)$. We know that covariance might be positive, negative, or zero - giving a idea of how quantities $Q$ and $Q^{\prime}$ are related. An important special case is given by probabilistically independent measurements of $Q$ and $Q^{\prime}$, defined by $\mathcal{S}\left(f(Q) \bullet g\left(Q^{\prime}\right)\right)=\mathcal{S}(f(Q)) \bullet \mathcal{S}\left(g\left(Q^{\prime}\right)\right)$ for all states $\mathcal{S}$.

defined by the averaging integral 


\section{ACKNOWLEDGEMENTS}

I thank Vadim Batitsky for numerous discussions relating to the foundational problems of measurement and for his valuable suggestions regarding several conceptual issues plaguing the earlier versions of this work.

\section{REFERENCES}

[1] Černý, M., Rada, M. (2011). On the possibilistic approach to linear regression with rounded or interval-censored data. Measurement Science Review 11 (2), 34-40.

[2] Domotor, Z., Batitsky, V. (2008). The analytic versus representational theory of measurement. Measurement Science Review 8, 129-146.

[3] Domotor, Z., Batitsky, V. (2009). Measurement, information channels, and discretization: Exploring the links. Measurement Science Review 9 (6), 134-161.

[4] Domotor, Z., Batitsky, V. (2010). An algebraic-analytic framework for measurement theory. Measurement Journal 43 (9), $1142-1164$.

[5] Hegeduš, H., Mostarac, P., Malaric, R. (2011). Comparison of RMS value measurement algorithms of non-coherent sampled signals. Measurement Science Review 11 (3), 79-84.
[6] Kakihara, Y. (1999). Abstract methods in information theory. World Scientific, Singapore.

[7] Kosarevsky, S.V., Latypov, V.N. (2012). Practical procedure for position tolerance uncertainty determination via Monte-Carlo error propagation. Measurement Science Review 12 (1), 1-7.

[8] Krantz, D.H., Luce, R.D., Suppes, P., Tversky, A. (1971). Foundations of measurement. Volume I: Additive and polynomial representations. Academic Press, New York.

[9] Morawski, R.Z., (1994). Unified approach to measurand reconstruction. IEEE Transaction on Instrumentation and Measurement 43, 226-231.

[10] Pratt, V.R. (1999). Chu spaces: Notes for school on category theory and applications. Technical report, University of Coimbra, Coimbra, Portugal. Available at http://boole.stanford.edu/pub/coimbra.pdf

[11] Sinha, V.P. (2010). Symmetries and groups in signal processing: An introduction. Springer, New York.

[12] Takesaki, M. (2002). Theory of operator algebra. Volume I. Springer, New York.

Received September 2, 2012. Accepted November 30, 2012. 